\title{
An Axiomatization of the Category of Petri Net Computations
}

\author{
VLADIMIRO SASSONE \\ BRICS* - Computer Science Department, University of Aarhus \\ Received 6 March 1997
}

\begin{abstract}
We introduce the notion of strongly concatenable process as a refinement of concatenable processes (Degano et al. 1996) which can be expressed axiomatically via a functor $Q$ (_) from the category of Petri nets to an appropriate category of symmetric strict monoidal categories, in the precise sense that, for each net $N$, the strongly concatenable processes of $N$ are isomorphic to the arrows of $Q(N)$. In addition, we identify a coreflection right adjoint to $Q(-)$ and characterize its replete image, thus yielding an axiomatization of the category of net computations.
\end{abstract}

\section{Introduction}

Petri nets, introduced by C.A. Petri in (Petri 1962) (see also Petri 1973; Reisig 1985), are unanimously considered one of the most representative models for concurrency, since they are a fairly simple and natural model of concurrent and distributed computations. However, Petri nets are, in our opinion, not yet completely understood.

Among the semantics proposed for Petri nets, a relevant role is played by the various notions of process (Petri 1977; Goltz and Reisig 1983; Best and Devillers 1987), whose merit is to provide a faithful account of computations involving many different transitions and of the causal connections between the events occurring in a computation. However, process models, at least in their standard forms, fail to bring to the foreground the algebraic structure of nets and their computations. Since such a structure is relevant to the understanding of nets, they fail, in our view, to give a comprehensive account of net behaviours.

The idea of looking at nets as algebraic structures (Reisig 1985; Nielsen et al. 1981; Winskel 1984; Winskel 1987; Brown and Gurr 1990; Brown et al. 1991) has been given an original interpretation by considering monoidal categories as a suitable framework (Meseguer and Montanari 1990). In fact, in (Meseguer and Montanari 1990; Degano et al. 1996) the authors have shown that the semantics of Petri nets can be understood in terms of symmetric monoidal categories - where objects are states, arrows processes, and the tensor product and the arrow composition model, respectively, the operations of parallel and sequential composition of processes. In particular, (Degano et al. 1996) introduced concatenable processes - a simple variation of Goltz-Reisig processes (Goltz and Reisig 1983) on which sequential composition can be defined - and structured the concatenable processes of a Petri net $N$ as the arrows of the symmetric

\footnotetext{
* Basic Research in Computer Science, Centre of the Danish National Research Foundation.

The author was supported by EU Human Capital and Mobility grant ERBCHBGCT 920005.
} 
strict monoidal category $P(N)$. This construction complements the process-oriented view of net computations by explaining their underlying algebra; more precisely, it yields an axiomatization of the causal behaviour of a net as an essentially algebraic theory, and thus provides a unification of the process and the algebraic view of net behaviours.

However, also this construction is somehow unsatisfactory, since it is not functorial. More strongly, given a morphism between two nets, i.e., a simulation between them, it may not be possible to identify a corresponding monoidal functor between the respective categories of computations. This fact, besides showing that our understanding of the algebraic structure of Petri nets is still incomplete, prevents us from identifying the category (of the categories) of net computations, i.e., from axiomatizing the behaviour of Petri nets 'in the large'.

This paper presents an analysis of this issue and a solution based on the new notion of strongly concatenable processes, introduced in Section 4. These are a slight refinement of concatenable processes which are still rather close to the standard notion of process: they are Goltz-Reisig processes whose minimal and maximal places are equipped with a linear ordering. In the paper we show that, similarly to concatenable processes, also this new notion can be axiomatized as an algebraic construction on $N$ by providing an abstract symmetric strict monoidal category $Q(N)$ whose arrows are in one-to-one correspondence with the strongly concatenable processes of $N$. The category $Q(N)$ constitutes our proposed axiomatization of the behaviour of $N$ in categorical terms.

Corresponding directly to the linear ordering of pre- and post-sets which characterizes strongly concatenable processes, the key feature of $Q\left(_{-}\right)$is that, differently from $\mathcal{P}\left({ }_{-}\right)$, it associates to the net $N$ a monoidal category whose objects form a free non-commutative monoid. The reason for renouncing to commutativity when passing from $P\left({ }_{-}\right)$to $Q\left(_{-}\right)$, a choice that at first may seem odd, is explained in Section 2, where the following negative result is proved:

under very reasonable assumptions, no mapping from nets to symmetric strict monoidal categories whose monoids of objects are commutative can be lifted to a functor, since there exists a morphism of nets which cannot be extended to a monoidal functor between the appropriate categories.

Thus, abandoning the commutativity of the monoids of objects and considering strings as explicit representatives of multisets, i.e., considering strongly concatenable processes, seem to be a choice forced upon us by the aim of a functorial algebraic semantics of nets. As a consequence of this choice, any transition of $N$ has many corresponding arrows in $Q(N)$, actually one for each linearization of its pre-set and of its post-set. However, such arrows are 'related' to each other by a naturality condition, in the precise sense that, when collected together, they form a natural transformation between appropriate functors. This naturality axiom is the second relevant feature of $Q(-)$ and it is actually the key for keeping the computational interpretation of the new category $Q(N)$, i.e., the strongly concatenable processes of $N$, surprisingly close to that of $P(N)$, i.e., the concatenable processes of $N$, and to the classic notion of net processes.

Concerning our main issue, viz. functoriality, in Section 3 we introduce a category SPetriCat of symmetric strict monoidal categories with free non-commutative monoids of objects, called symmetric Petri categories, whose arrows are equivalence classes - accounting for our view of strings as representatives of multisets - of those symmetric strict monoidal functors which preserve some further structure related to nets, and we show that $Q(-)$ is a functor from Petri, a 
rich category of nets introduced in (Meseguer and Montanari 1990), to SPetriCat. In addition, we prove that $Q\left({ }_{-}\right)$has a coreflection right adjoint $\mathcal{N}\left({ }_{-}\right)$:SPetriCat $\rightarrow$ Petri. This implies, by general reasons, that Petri is equivalent to an easily identified coreflective subcategory of SPetriCat, namely the replete image of $Q(-)$. The category SPetriCat, together with the functors $Q(-)$ and $\mathcal{N}(-)$, constitutes our proposed axiomatization ('in the large') of Petri net computations in categorical terms.

Although this contribution is a first attempt towards the aims of a functorial algebraic semantics for nets and of an axiomatization of net behaviours 'in the large', we think that the results given here help to deepen the understanding of the subject. We remark that the refinement of concatenable processes into strongly concatenable processes is similar and comparable to the one which brought from Goltz-Reisig processes to them, and that the result of Section 2 makes strongly concatenable processes 'unavoidable' if a functorial construction is desired. In addition, from the categorical viewpoint, our approach is quite natural, since it is the one which simply observes that multisets are equivalence classes of strings and then takes into account the categorical paradigm, following which one always prefers to add suitable isomorphisms between objects rather than considering explicitly equivalence classes of them. Finally, concerning the use of category theory in semantics, and in particular in this paper, it may be appropriate to observe here that the categorical framework made it possible to discover and amend a significant 'anomaly' of concatenable processes which, although of general relevance, would have not been easily noticed in other frameworks.

This paper is a full and extended version of (Sassone 1995). Some preliminary related results appear also in the author's thesis (Sassone 1994).

$\underline{\text { Notation. }}$. Given a category C, we denote the composition of arrows in C by the usual symbol _ ${ }_{-}$and follow the usual right to left order. The identity of $c \in \mathrm{C}$ is written as $i d_{c}$. However, we make the following exception. When dealing with a category in which arrows are meant to represent computations, in order to stress this, we write arrow composition from left to right, i.e., in the diagrammatic order, and we denote it by _ ; _. Moreover, when no ambiguity arises, $i d_{c}$ is simply written as $c$. We shall use SSMC to indicate the category of (small) symmetric strict monoidal categories and symmetric strict monoidal functors. Since the monoidal categories considered in the paper are always strict monoidal and (non-strictly) symmetric, we may sometimes omit to mention all the attributes without causing misunderstandings.

The reader is referred to (MacLane 1971) for the categorical concepts used in the paper. The basic definitions concerning symmetric strict monoidal categories are summarized in Appendix A.

Acknowledgments. I wish to thank José Meseguer and Ugo Montanari to whom I am indebted for several discussions on the subject. Thanks to Mogens Nielsen, Claudio Hermida, Jaap van Oosten, and the anonymous referees for their valuable comments on an early version of this paper.

\section{Concatenable Processes}

In this section we recall the notion of concatenable process (Degano et al. 1996) and we give the definitions which will be used in the rest of the paper.

Notation. Given a function $v$ from a set $S$ to the set of natural numbers $\omega$, its support is the subset of $S$ consisting of those elements $s$ such that $v(s)>0$. We denote by $S^{\oplus}$ the set of finite multisets of $S$, i.e., the set of all functions from $S$ to $\omega$ with finite support. We shall represent a finite multiset $\nu \in S^{\oplus}$ as a formal 
sum $\bigoplus_{i \in I} n_{i} s_{i}$ where $\left\{s_{i} \mid i \in I\right\}$ is the support of $v$ and $n_{i}=v\left(s_{i}\right)$, i.e., as a sum whose summands are all nonzero. Recall that $S^{\oplus}$ is a commutative monoid, actually the free commutative monoid on $S$, under the operation of multiset union with unit element the empty multiset 0 .

Definition 1.1. (Petri Nets) A Place/Transition Petri net is a structure $N=\left(\partial_{N}^{0}, \partial_{N}^{1}: T_{N} \rightarrow S_{N}^{\oplus}\right)$, where $T_{N}$ is a set of transitions, $S_{N}$ is a set of places, $\partial_{N}^{0}$ and $\partial_{N}^{1}$ are functions.

A morphism of nets from $N_{0}$ to $N_{1}$ is a pair $f=\left\langle f_{T}, f_{S}\right\rangle$, where $f_{T}: T_{N_{0}} \rightarrow T_{N_{1}}$ is a function and $f_{S}: S_{N_{0}}^{\oplus} \rightarrow S_{N_{1}}^{\oplus}$ is a monoid homomorphism, that respects source and target, i.e., the two squares obtained by choosing the upper or lower arrows in the parallel pairs of the diagram below commute.

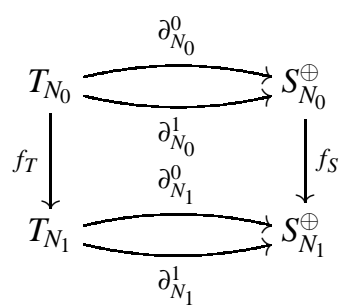

This, with the obvious componentwise composition of morphisms, defines the category Petri of nets.

Thus, according to Definition 1.1, a Petri net is precisely as a graph whose set of nodes is a free commutative monoid, i.e., the set of finite multisets on a given set of places. The source and target of an arc, here called a transition, are meant to represent, respectively, the markings consumed and produced by the firing of the transition.

In the noninterleaving approaches to concurrency, it is common to describe computations in terms of partial orderings representing the causal relationships between event occurrences. In the case of nets, this is fruitfully formalized through the following notion of process.

Definition 1.2. (Process Nets and Processes) A process net is a finite, acyclic net $\Theta$ such that

(i) for all $t \in T_{\Theta}, \partial_{\Theta}^{0}(t)$ and $\partial_{\Theta}^{1}(t)$ are sets (as opposed to multisets);

(ii) for all pairs $t_{0} \neq t_{1} \in T_{\Theta}, \partial_{\Theta}^{i}\left(t_{0}\right) \cap \partial_{\Theta}^{i}\left(t_{1}\right)=\varnothing$, for $i=0,1$.

Given $N \in$ Petri, a process of $N$ is a morphism $\pi: \Theta \rightarrow N$, where $\Theta$ is a process net and $\pi$ is a net morphism which maps places to places (as opposed to morphisms which map places to markings).

The constraint on $\pi$ in the definition above means that process morphisms map a single component of the process net to a single component of $N$; otherwise said, processes are nothing but labelings of $\Theta$ - which in turn, as implied by the constraints on it, is essentially a partial ordering of transitions - with appropriate elements of $N$.

In order to define processes at the right level of abstraction, we need to make some identifications and consider as identical process nets which are isomorphic. Precisely, we shall make no distinction between processes $\pi: \Theta \rightarrow N$ and $\pi^{\prime}: \Theta^{\prime} \rightarrow N$ for which there exists an isomorphism $\varphi: \Theta \rightarrow \Theta^{\prime}$ of the underlying process nets such that $\pi^{\prime} \circ \varphi=\pi$.

Example 1.3. Figure 1, in which we use the standard graphical representation of nets with circles for places, boxes for transitions, and directed arcs for sources and targets, shows a net $N$ 


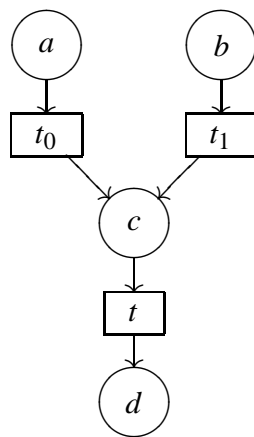

$N$
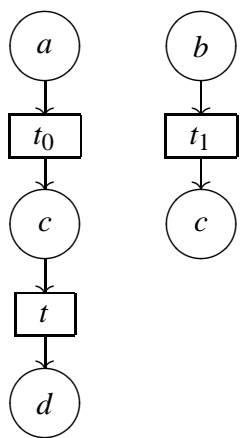
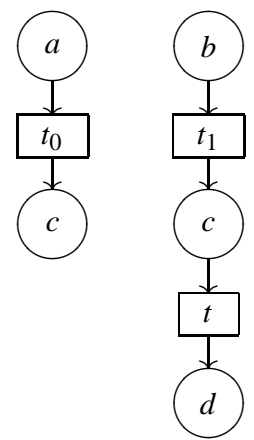

$\pi^{\prime}$

Figure 1. A net $N$ and two of its processes

with $S_{N}=\{a, b, c, d\}$ and $T_{N}=\left\{t_{0}: a \rightarrow c, t_{1}: b \rightarrow c, t: c \rightarrow d\right\}$ and two of its processes. A process $\pi$ of $N$ is represented implicitly by labeling each element $x$ of its process net by $\pi(x)$, the (unique) element of $N$ it corresponds to. The picture also makes clear the distinct roles of the two components of processes: the morphism $\pi$ identifies which elements of $N$ are involved in the computation, whilst the process net $\Theta$ serves to describe their causal links.

It is worth observing that $\pi$ and $\pi^{\prime}$, although are based on the same process net, describe computations which are rather different from the causal point of view: in $\pi$ the occurrence of $t$ depends on $t_{0}$, whereas it depends on $t_{1}$ in $\pi^{\prime}$. In particular, $\pi$ and $\pi^{\prime}$ are not isomorphic.

As already mentioned in the introduction, processes explain only the meaning of single computations, and fail to describe the algebraic structure of the space of behaviours as a whole. On the contrary, following well-established ideas in semantics, it would certainly be desirable to have an algebra of processes that singles out the basic building blocks of such a space, their compositional properties, and the laws they are governed by. Clearly enough, the bulk of such an algebra should consist of the operations of sequential and parallel composition. Unfortunately, due to the inherent ambiguity on the identity of the instances of places that occur in processes (the so-called tokens), process concatenation is not a well defined operation. In the example above, for instance, both $\pi$ and $\pi^{\prime}$ are decomposable into a concatenation of the same two processes, namely the one that runs $t_{0}$ and $t_{1}$ in parallel, followed by the one that runs $t$ : the difference between them resides entirely in the choice of one of the two possible sources for $t$. This means that concatenation must handle the flow of causal dependencies between subprocesses; and since such a flow is regulated down to the level of tokens, process concatenation must be concerned with merging tokens rather than places. It is therefore necessary to disambiguate the identity of all the tokens a process offers as possible 'concatenation points'.

The solution proposed in (Degano et al. 1996) is the following notion concatenable processes. As a matter of notation, we shall use $\min (\Theta)$ and $\max (\Theta)$ denote the 'minimal' and 'maximal' places of a process net $\Theta$, i.e., the places of $\Theta$ belonging respectively to the post-set and to the pre-set of no transition.

Definition 1.4. (Concatenable Processes) A concatenable process of $N$ is a triple $C P=(\pi, \ell, L)$ where

$-\pi: \Theta \rightarrow N$ is a process of $N$; 
- $\ell$ is a family of total orderings $\ell_{b}$ of $\pi_{S}^{-1}(b) \cap \min (\Theta)$, for $b \in S_{N}$.

- $L$ is a family of total orderings $L_{b}$ of $\pi_{S}^{-1}(b) \cap \max (\Theta)$, for $b \in S_{N}$.

Two concatenable processes are isomorphic, and thus identified, if their underlying processes are isomorphic via an isomorphism that respects all the orderings.

It is now easy to define an operation of concatenation of concatenable processes, whence their name. We can associate a source and a target in $S_{N}^{\oplus}$ to any concatenable process $C P$, namely by taking the image through $\pi$ of, respectively, $\min (\Theta)$ and $\max (\Theta)$, where $\Theta$ is the underlying process net of $C P$. Then, the concatenation of concatenable processes $\left(\pi_{0}: \Theta_{0} \rightarrow N, \ell_{0}, L_{0}\right): u \rightarrow v$ and $\left(\pi_{1}: \Theta_{1} \rightarrow N, \ell_{1}, L_{1}\right): v \rightarrow w$ is realized by merging the maximal places of $\Theta_{0}$ and the minimal places of $\Theta_{1}$ using $\pi_{0}, \pi_{1}$, and the orderings $L_{0}$ and $\ell_{1}$ to match those places one-to-one (cf. Figure 2). Under this operation of sequential composition, the concatenable processes of $N$ form a category $\mathcal{C P}(N)$ with identities those processes consisting only of places, which therefore are both minimal and maximal, and such that $\ell=L$.

Concatenable processes admit also a tensor operation $\otimes$ which can be represented as putting two processes side by side and extending their orderings in such a way that the places of the process on the left precede those of the one on the right (cf. Figure 2). The algebra of (concatenable) processes so obtained enjoys some interesting properties that suggest a connection to symmetric monoidal categories, as for instance the facts that parallel composition is essentially but not strictly commutative, and that whenever $\left(C P_{0} ; C P_{0}^{\prime}\right) \otimes\left(C P_{1} ; C P_{1}^{\prime}\right)$ is defined it coincides with $C P_{0} \otimes C P_{1} ; C P_{0}^{\prime} \otimes C P_{1}^{\prime}$. In fact, it easy to prove that the concatenable processes consisting only of places form the category of symmetries which make $C P(N)$ into a symmetric strict monoidal category. Then, in order to find an axiomatization of the algebra, i.e., to understand in abstract terms both processes and the laws that rule their composition, one can proceed trying to capture $C \mathcal{P}(N)$ by means of (categorical) universal constructions. This is the purpose of the following improved definition of the category $\mathcal{P}(N)$, whose equivalence with the original one in (Degano et al. 1996) has been proved in (Sassone 1996).

Definition 1.5. (The Category $\mathscr{P}(N)$ ) The category $\mathscr{P}(N)$ is the monoidal quotient (see Appendix A) of $\mathcal{F}(N)$, the free symmetric strict monoidal category generated by $N$, obtained by imposing the axioms

$$
\begin{aligned}
c_{a, b} & =i d_{a \oplus b} & & \text { if } a, b \in S_{N} \text { and } a \neq b, \\
s ; t ; s^{\prime} & =t & & \text { if } t \in T_{N} \text { and } s, s^{\prime} \text { are symmetries, }
\end{aligned}
$$

where $c, i d, \otimes$, and $\_;_{-}$are, respectively, the symmetry isomorphism, the identities, the tensor product, and the composition of $\mathcal{F}(N)$.

The main result of (Degano et al. 1996) is that $\mathcal{P}(N)$ offers a precise, abstract description of the algebra of concatenable processes of $N$.

Proposition 1.6. (Concatenable Processes vs. $\left.P\left({ }_{-}\right)\right) \mathcal{C} P(N)$ and $P(N)$ are isomorphic in SSMC.

Example 1.7. Figure 2 shows a concatenable process $\pi$ of the net $N$ of Figure 1 that corresponds to the arrow $t_{0} \otimes t_{1} ; t \otimes i d_{c}$ of $\mathcal{P}(N)$. The orderings $\ell$ and $L$, omitted whenever irrelevant, are represented in the obvious way by labeling places with natural numbers. To exemplify the algebra of processes of $N, \pi$ is expressed as parallel $\left({ }_{-} \otimes_{-}\right)$and sequential $\left({ }_{-} ;_{-}\right)$composition of simpler 


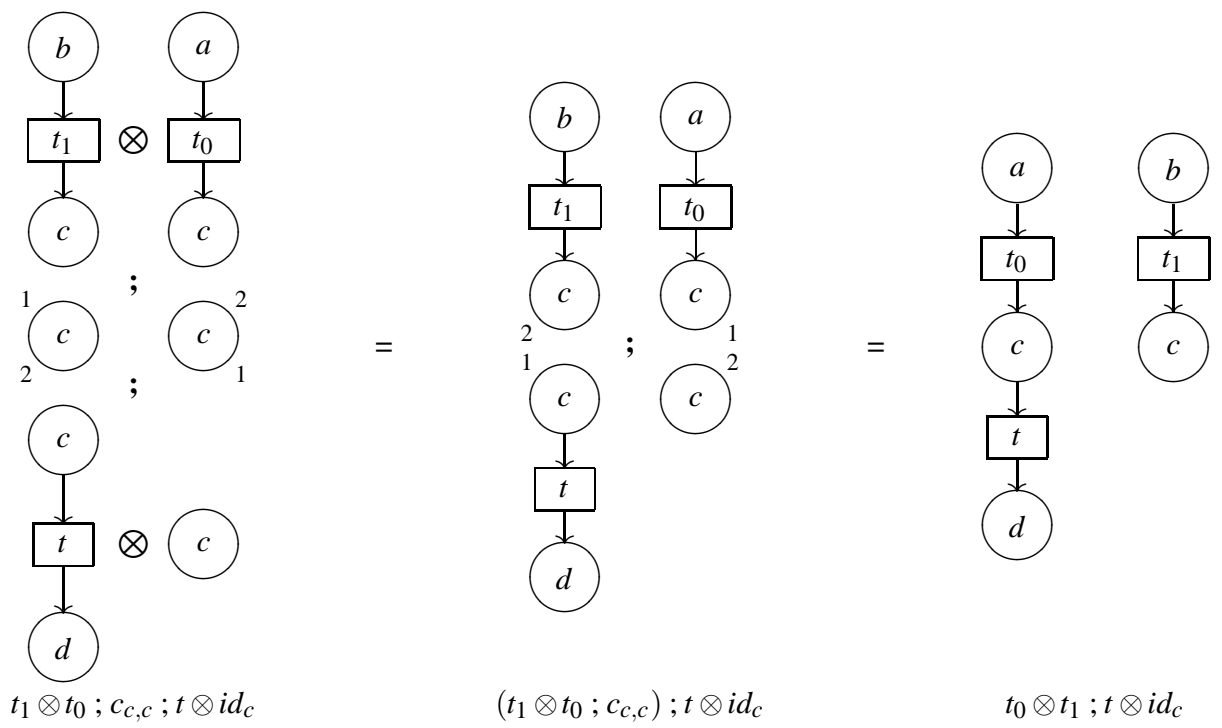

Figure 2. The concatenable process $\pi=t_{0} \otimes t_{1} ; t \otimes i d_{c}$ of the net $N$ of Figure 1

processes. Such operations are matched precisely by operations and axioms of $\mathcal{P}(N)$, and this is the essence of Proposition 1.6 above.

The symmetries of $\mathcal{P}(N)$, of course, correspond one-to-one to those of $\mathcal{C} P(N)$ which, as already mentioned, are the processes consisting only of places. For instance, $c_{c, c}: c \oplus c \rightarrow c \oplus c$ represents the (unique up to isomorphism) process that inverts in its 'output' the order of the two 'input' tokens. This simple example illustrates that the role of the symmetries in process terms is that of regulating the flow of causality between subprocesses by permuting tokens appropriately. In fact, such permutations yield exactly the 'exchanges' of causes needed to gain control over the operation of process concatenation. It is a rather interesting observation, in our opinion, that the axioms of symmetries in monoidal categories, together with those of $\mathcal{P}(N)$, capture this precisely enough. With this understanding of symmetries, we can read the axioms of Definition 1.5 as follows: (1) tokens that are instances of different places cannot be permuted, since they differ structurally, not just for their causal histories; and (2) permuting tokens in the pre- and in the post-set of a single transition $t$ has no effect whatsoever on causality, since it does not change causes or effects of $t$.

Concerning the generators of $\mathcal{P}(N)$, i.e., the transitions of $N$, under the correspondence of Proposition 1.6 the arrow $t: u \rightarrow v$ for $t \in T_{N}$ represents the unique concatenable process $C P: u \rightarrow v$ consisting of the single transition $t$. Observe that there is a unique such process since the labeling of places in $C P$ are irrelevant in this case: they all yield isomorphic processes. This fact is directly reflected by the second axiom in the definition of $P(N)$.

\section{A Negative Result about Functoriality}

Among the primary requirements usually imposed on constructions like $\mathcal{P}(-)$ there is that of functoriality. One of the reasons supporting a categorical treatment of semantics is the need for specifying further the structure of the systems under analysis by considering morphisms be- 
tween them which determine, e.g., how the given systems simulate each other. This, in turn, means choosing precisely what the relevant (behavioural) structure of the systems is. It is therefore clear that such morphisms should be preserved at the semantic level. In the case of $\mathcal{P}(-)$, this means that whenever $N$ can be mapped to $N^{\prime}$ via a morphism $f$, which by the very definition of net morphisms implies that $N$ can be simulated by $N^{\prime}$, there must be a way, namely $P(f)$, to map the concatenable processes of $N$ in those of $N^{\prime}$, respecting their algebraic structure. However, this is not the case: there are net morphisms which cannot be extended to symmetric monoidal functors between the respective categories of processes. The problem, as illustrated by the following example, is due to the particular shape of the symmetries of $\mathcal{P}(N)$ which, on the other hand, is exactly what makes $\mathcal{P}(N)$ capture quite precisely the notion of processes of $N$.

Example 2.1. ( $\left(P\left(_{-}\right)\right.$cannot be a functor) Consider the nets $N$ and $\bar{N}$ in the picture below. We have $S_{N}=\left\{a_{0}, a_{1}, b_{0}, b_{1}\right\}$ and $T_{N}$ consisting of the transitions $t_{0}: a_{0} \rightarrow b_{0}$ and $t_{1}: a_{1} \rightarrow b_{1}$, while $S_{\bar{N}}=\left\{\bar{a}_{1} \bar{b}_{0}, \bar{b}_{1}\right\}$ and $T_{\bar{N}}$ contains $\bar{t}_{0}: \bar{a} \rightarrow \bar{b}_{0}$ and $\bar{t}_{1}: \bar{a} \rightarrow \bar{b}_{1}$.

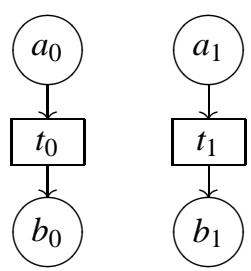

$N$

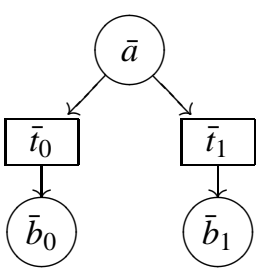

$\bar{N}$

Consider now the net morphism $f$ where $f_{T}\left(t_{i}\right)=\bar{t}_{i}, f_{S}\left(a_{i}\right)=\bar{a}$ and $f_{S}\left(b_{i}\right)=\bar{b}_{i}$, for $i=0,1$. We claim that $f$ cannot be extended to a monoidal functor $\mathcal{P}(f)$ from $\mathcal{P}(N)$ to $\mathcal{P}(\bar{N})$. Suppose in fact that $\mathrm{F}$ is such an extension. Then, we must have $\mathrm{F}\left(t_{0} \otimes t_{1}\right)=\mathrm{F}\left(t_{0}\right) \otimes \mathrm{F}\left(t_{1}\right)=\bar{t}_{0} \otimes \bar{t}_{1}$. Moreover, since $t_{0} \otimes t_{1}=t_{1} \otimes t_{0}$, we must also have

$$
\bar{t}_{0} \otimes \bar{t}_{1}=\mathrm{F}\left(t_{1} \otimes t_{0}\right)=\bar{t}_{1} \otimes \bar{t}_{0},
$$

which is impossible, since the leftmost and the rightmost terms above are different processes in $P(\bar{N})$, as follows from Definition 1.5.

Formally speaking, the problem is that the category of symmetries sitting inside $P(N)$, say $\operatorname{Sym}_{N}$, is not free on $N$. Clearly, this is due to first axiom of Definition 1.5, a conditional axiom with a negative premise. To make things worse, the theory illustrated extensively in (Degano et al. 1996; Sassone 1994) makes it clear that, in order for $\mathcal{P}(N)$ to maintain its interesting computational meaning, such an axiom is strictly needed. Moreover, it is easy to verify that as soon as one imposes further axioms on $P(N)$ which guarantee to get a functor, one annihilates all the symmetries and, therefore, destroys the ability of $P(N)$ to deal with causality. It is also important to observe that it would be definitely meaningless to try to overcome the problem simply by removing from Petri the morphisms which 'behave badly': the morphism $f$ of Example 2.1, for instance, is clearly a simulation and, as such, it should definitely be allowed by any serious attempt to formulate a definition of net morphisms. Finally, also the natural idea of looking for non strict monoidal functors (Eilenberg and Kelly 1966) as semantic counterparts of net morphisms does not lead anywhere, as implicitly shown again by Example 2.1. 
In other words, there does not seem to be an easy and satisfactory solution to the functoriality problem for $\mathcal{P}\left({ }_{-}\right)$. The following proposition shows that the problem illustrated in Example 2.1 is serious, actually deep enough to prevent any naive modification of $\mathcal{P}\left({ }_{-}\right)$to be functorial.

Proposition 2.2. (No simple variation of $\left.P_{(-}\right)$can be a functor) Let $\left.X_{(-}\right)$be a function which assigns to each net $N$ a symmetric strict monoidal category whose monoid of objects is commutative and contains $S_{N}$, the places of $N$. Suppose further that the group of symmetries at any object of $x(N)$ is finite. Finally, suppose that there exists a net $N$ with a place $a \in S_{N}$ such that, for each $n \geq 1$, we have that the component at $(n a, n a)$ of the symmetry isomorphism of $x(N)$ is not an identity.

Then, there exists a Petri net morphism $f: N_{0} \rightarrow N_{1}$ which cannot be extended to a symmetric strict monoidal functor from $X\left(N_{0}\right)$ to $X\left(N_{1}\right)$.

Proof. The key of the proof is the following observation about monoidal categories. Let $\mathrm{C}$ be a symmetric strict monoidal category with symmetry isomorphism $c$. Then, for all $a \in \mathrm{C}$ and for all $n \geq 1$, we have $\left(c_{a,(n-1) a}\right)^{n}=i d$, where, in order to simplify the notation, throughout the proof we write $n a$ and $c_{x, y}^{n}$ to denote, respectively, the tensor product of $n$ copies of $a$, with $0 a=e$, and the sequential composition of $n$ copies of $c_{x, y}$. To show that the above identity holds, consider for $i=1, \ldots, n$ the functor $\mathrm{F}_{i}$ from $\mathrm{C}^{n}$, the cartesian product of $n$ copies of $\mathrm{C}$, to $\mathrm{C}$ defined as follows.

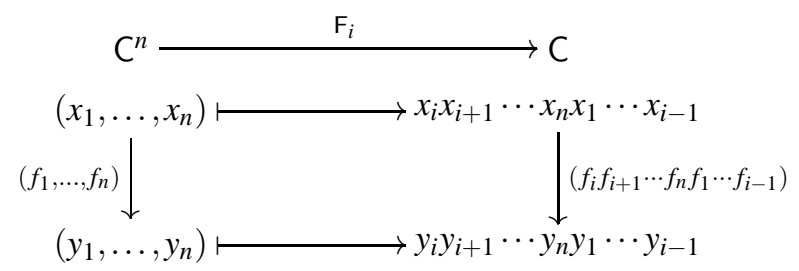

Moreover, consider the natural transformations $\phi_{i}: \mathrm{F}_{i} \dot{\rightarrow} \mathrm{F}_{i+1}, i=1, \ldots, n-1$ and $\phi_{n}: \mathrm{F}_{n} \rightarrow \mathrm{F}_{1}$ whose components at $x_{1}, \ldots, x_{n}$ are, respectively, $c_{x_{i}, x_{i+1} \cdots x_{n} x_{1} \cdots x_{i-1}}$ and $c_{x_{n}, x_{1} \cdots x_{n-1}}$. Finally, let $\phi$ be the sequential composition of $\phi_{1}, \ldots, \phi_{n}$. Then, $\phi$ is a natural transformation $x_{1} \cdots x_{n} \dot{\rightarrow} x_{1} \cdots x_{n}$ built up only from components of $c$. From the Kelly-MacLane coherence theorem (MacLane 1963; Kelly 1964) (see also Appendix A) we know that there is at most one natural transformation consisting only of identities and components of $c$, and since the identity of $\mathrm{F}_{1}$ is one such transformation, we have that $\phi=i d_{\mathrm{F}_{1}}$. Then, instantiating each variable with $a$, we obtain $\left(c_{a,(n-1) a}\right)^{n}=i d_{n a}$, as required.

It is now easy to conclude the proof. Let $N^{\prime}$ be a net with $a \in S_{N^{\prime}}$ such that, for each $n \geq 1$, we have $c_{n a, n a}^{\prime} \neq i d$, where $c^{\prime}$ is the symmetry natural isomorphism of $x\left(N^{\prime}\right)$, let $N$ be a net with two distinct places $a$ and $b$ and with no transitions, and let $c$ be the symmetry natural isomorphism of $x(N)$. Since the group of symmetries at $a b$ is finite, there is a cyclic subgroup generated by $c_{a, b}$, i.e., there exists $k \geq 1$, the order of the subgroup, such that $\left(c_{a, b}\right)^{k}=i d$ and $\left(c_{a, b}\right)^{n} \neq i d$ for any $1 \leq n<k$.

Let $p$ be any prime number greater than $k$. We claim that the net morphism $f: N \rightarrow N^{\prime}$, where $f_{T}$ is the (unique) function $\varnothing \rightarrow T_{N^{\prime}}$ and $f_{S}$ is the monoid homomorphism such that $f_{S}(b)=(p-1) a$ and $f_{S}(a)=a$, cannot be extended to a symmetric strict monoidal functor $\mathrm{F}: \chi(N) \rightarrow \chi\left(N^{\prime}\right)$. In fact, from the first part of this proof, we know that $\left(c_{a,(p-1) a}^{\prime}\right)^{p}=i d$. Moreover, by general results of group theory, the order of the cyclic subgroup generated by $c_{a,(p-1) a}^{\prime}$ must be a factor 
of $p$ and then, in this case, 1 or $p$. In other words, either $c_{a,(p-1) a}^{\prime}=i d$, or $\left(c_{a,(p-1) a}^{\prime}\right)^{n} \neq i d$ for all $1 \leq n<p$. If the second situation occurs, then we have that $\mathrm{F}\left(\left(c_{a, b}\right)^{k}\right)=i d$ and also that $\mathrm{F}\left(\left(c_{a, b}\right)^{k}\right)=\left(c_{\mathrm{F}(a), \mathrm{F}(b)}^{\prime}\right)^{k}=\left(c_{a,(p-1) a}^{\prime}\right)^{k} \neq i d$, i.e., $\mathrm{F}$ cannot exists. Thus, in order to conclude the proof, we only need to show that it follows from our hypothesis that $c_{a,(p-1) a}^{\prime} \neq i d$. For this, it is enough to observe that $c_{a,(p-1) a}^{\prime}=i d$ implies $c_{n a, n a}^{\prime}=i d$ for $n=p-1$, which is against our hypothesis on $N^{\prime}$. In fact, by the axioms of symmetries in monoidal categories (viz. the first of (6) in Appendix A) $c_{k a,(p-1) a}^{\prime}=a c_{(k-1) a,(p-1) a}^{\prime} ; c_{a,(p-1) a}^{\prime}(k-1) a$, whence it follows directly that $c_{(p-1) a,(p-1) a}^{\prime}=i d$.

The contents of the previous proposition may be further clarified by remarking that in the free category of symmetries on a commutative monoid $M$ there are infinite homsets. This means that dropping axiom $c_{a, b}=i d_{a \oplus b}$ in the definition of $\mathcal{P}(N)$ causes an 'explosion' in the structure of the symmetries. More precisely, if we omit that axiom, we can find some object $u$ such that the group of symmetries on $u$ has infinite order. Of course, since symmetries represent causality, and as such they are integral parts of processes, this makes the category so obtained completely useless for the applications we have in mind.

The hypotheses of Proposition 2.2 can be certainly weakened in several ways, at the expense of complicating the proof. However, we avoided such complications since the conditions stated above are already weak enough if one wants to regard $x(N)$ as a category of processes of $N$. In fact, since places represent the atomic bricks of which states are built, one needs to consider them in $x(N)$. Since symmetries regulate the 'flow of causality', there will be $c_{n a, n a}$ different from the identity, and since in a computation we can have only finitely many 'causality streams', there should not be categories of processes with infinite groups of symmetries. Therefore, the given result means that there is no chance to have a functorial construction along the lines of $\mathcal{P}(N)$ for the category of processes of a Petri net $N$ if the objects form a commutative monoid.

\section{The Category $Q(N)$}

In this section we introduce the symmetric strict monoidal category $Q(N)$ which is meant to represent the processes of the Petri net $N$ and which supports a functorial construction. This will allow us to characterize the category of categories of net behaviours, i.e., to axiomatize the behaviour of nets 'in the large'. Observe that although (Meseguer and Montanari 1990) and (Degano et al. 1996) clarify how the behaviour of a single net can be captured by a symmetric strict monoidal category, due to the lack of functoriality of $\mathcal{P}\left({ }_{-}\right)$, they cannot propose a categorical semantics 'in the large' for net causal behaviours.

Proposition 2.2 shows that, necessarily, there is a price to be payed. Here, the idea is to renounce to the commutativity of the monoids of objects. More precisely, the arrows of $Q(N)$ are built over $S y m_{N}^{*}$, the free category of symmetries on the set $S_{N}$ of places of $N$. This implies that objects form the free non-commutative monoid on $S_{N}$, and that symmetries are precisely the permutations of such objects. Looking at such strings of places as concrete realizations of multisets, the natural way to represent the transitions of $N$ is by replicating them, one copy of a transition for each choice of representatives of its source and target multisets. In other terms, the generators of $Q(N)$ are not transitions: more concretely, they are instances of transitions acting on the chosen representatives of states. Thus, each transition of $N$ has many corresponding arrows 
in $Q(N)$; in order to guarantee that $Q(N)$ remains close to the category $\mathcal{P}(N)$ of concatenable processes, therefore, it is necessary to link together all such arrows. This is achieved by means of a 'naturality' condition, allowing us to prove in Section 4 that the arrows of $Q(N)$ correspond to Goltz-Reisig processes in which the minimal and the maximal places are linearly ordered.

Similarly to $S y m_{N}$, the category $S y m_{N}^{*}$ serves a double purpose. While from the categorical point of view it provides the symmetry isomorphism of a symmetric monoidal category, from a semantic perspective it regulates the flow of causal dependency. It should be noticed, however, that here the point of view is slightly more concrete than, though not essentially dissimilar from, the case of $\operatorname{Sym}_{N}$. Computationally, a symmetry in $Q(N)$ must be interpreted as a 'reorganization' of the tokens in the global state of the net which, when reorganizing tokens belonging to the same place, yields a exchange of causes exactly as $S_{y} m_{N}$ does for $\mathcal{P}(N)$. In general, as will become clear later, an arrow of $Q(N)$ should be thought of as the realization of a processes of $N$ corresponding to an actual representation of its source and target states.

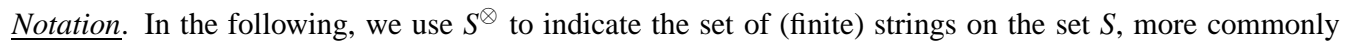
denoted by $S^{*}$. In the same way, we use $\otimes$ to denote string concatenation, while $\varepsilon$ denotes the empty string. As usual, for $u \in S^{\otimes}$, we indicate by $|u|$ the length of $u$ and by $u_{i}$ its $i$ th element. Moreover, we let $\mathcal{M}(u)$ denote the multiset of the elements of $S$ occurring in $u$.

Definition 3.1. (The category $Q(N)$ ) The category $Q(N)$, for $N$ a net in Petri, is obtained from the symmetric strict monoidal category freely generated from the places of $N$ and, for each transition $t$ of $N$, an arrow $t_{u, v}: u \rightarrow v$ for each pair of linearizations (as strings) $u$ and $v$ of the preand post- sets (multisets) of $t$, by quotienting modulo the axiom

$$
s ; t_{u^{\prime}, v}=t_{u, v^{\prime}} ; s^{\prime}, \quad \text { for } s: u \rightarrow u^{\prime} \text { and } s^{\prime}: v^{\prime} \rightarrow v \text { symmetries. }
$$

Explicitly, $Q(N)$ is (isomorphic to) the category C whose objects are the elements of $S_{N}^{\otimes}$ and whose arrows are generated by the inference rules

$$
\begin{aligned}
& \frac{u \in S_{N}^{\otimes}}{i d_{u}: u \rightarrow u \text { in } C} \quad \frac{u, v \text { in } S_{N}^{\otimes}}{c_{u, v}: u \otimes v \rightarrow v \otimes u \text { in } C} \quad \frac{t: \mathcal{M}(u) \rightarrow \mathcal{M}(v) \text { in } T_{N}}{t_{u, v}: u \rightarrow v \text { in } C} \\
& \frac{\alpha: u \rightarrow v \text { and } \beta: u^{\prime} \rightarrow v^{\prime} \text { in } C}{\alpha \otimes \beta: u \otimes u^{\prime} \rightarrow v \otimes v^{\prime} \text { in } C} \quad \frac{\alpha: u \rightarrow v \text { and } \beta: v \rightarrow w \text { in } C}{\alpha ; \beta: u \rightarrow w \text { in } C}
\end{aligned}
$$

modulo the axioms expressing that $\mathrm{C}$ is a strict monoidal category, namely,

$$
\begin{array}{rll}
\alpha ; i d_{v}=\alpha=i d_{u} ; \alpha & \text { and } \quad(\alpha ; \beta) ; \delta=\alpha ;(\beta ; \delta), \\
(\alpha \otimes \beta) \otimes \delta=\alpha \otimes(\beta \otimes \delta) & \text { and } \quad i d_{\varepsilon} \otimes \alpha=\alpha=\alpha \otimes i d_{\varepsilon}, \\
i d_{u} \otimes i d_{v}=i d_{u \otimes v} & \text { and } \quad\left(\alpha \otimes \alpha^{\prime}\right) ;\left(\beta \otimes \beta^{\prime}\right)=(\alpha ; \beta) \otimes\left(\alpha^{\prime} ; \beta^{\prime}\right),
\end{array}
$$

(the latter whenever the right-hand term is defined), plus the following axioms corresponding to axioms (6) expressing that $\mathrm{C}$ is symmetric with symmetry isomorphism $c$

$$
\begin{aligned}
c_{u, v \otimes w} & =\left(c_{u, v} \otimes i d_{w}\right) ;\left(i d_{v} \otimes c_{u, w}\right), \\
c_{u, u^{\prime}} ;(\beta \otimes \alpha) & =(\alpha \otimes \beta) ; c_{v, v^{\prime}} \quad \text { for } \alpha: u \rightarrow v, \beta: u^{\prime} \rightarrow v^{\prime}, \\
c_{u, v} ; c_{v, u} & =i d_{u \otimes v},
\end{aligned}
$$

and, finally, axiom $(\Phi)$. 
Observe that, while the first part of the previous definition gives $Q(N)$ in terms of universal constructions, its second part provides a completely axiomatic description of $Q(N)$ which can be useful in many contexts. In the following, we shall in fact use as definition of $Q(N)$ whichever version, constructive or axiomatic, is best suited to the actual application.

It is worth noticing that axiom $(\Phi)$ entails, as a particular case, the last axiom in the Definition 1.5 of $\mathcal{P}(N)$ - called axiom $(\Psi)$ in (Degano et al. 1996) - whenever it makes sense in $Q(N)$. In fact, axiom $(\Phi)$ asserts that any diagram in $Q(N)$ of the kind

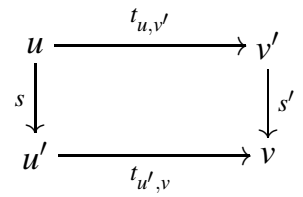

commutes. Then, fixing $u=u^{\prime}$ and $v=v^{\prime}$, and using the fact that symmetries are isomorphisms, one obtains precisely axiom $(\Psi)$. Exploiting this observation it is not difficult to show that there exists a quotient functor $Q(N) \rightarrow \mathcal{P}(N)$ in SSMC, and that $P(N)$ can be obtained from $Q(N)$ simply by enforcing commutativity on objects and the first axiom in Definition 1.5. Moreover, using $(\Phi)$ for $u \neq u^{\prime}$ or $u \neq u^{\prime}$, choosing $s^{\prime}=i d$, respectively $s=i d$, one proves that the interesting equation $s ; t_{u^{\prime}, v}=t_{u, v}$, respectively $t_{u, v^{\prime}} ; s^{\prime}=t_{u, v}$, holds in $Q(N)$.

A simple inspection of Definition 3.1 suggests that the category of symmetries of $Q(N)$, say $S y m_{N}^{*}$, is the free symmetric strict monoidal category on the set $S_{N}$. In fact, by definition, an arrow of $Q(N)$ is a symmetry if and only if the rule for transitions has never been used in its generation, and, moreover, axiom $(\Phi)$ - the only one introducing identifications not imposed by the definition of symmetric monoidal categories - has clearly no effect on such arrows. We shall now give a useful direct description of $S y m_{N}^{*}$ that, besides making explicit some of the structure of $Q(N)$, will play a relevant role in the development to come. Namely, we shall prove that $S y m_{N}^{*}$ is the category of permutations on $S_{N}$.

$\underline{\text { Notation. }}$. We use $\Pi(n)$ to denote the symmetric group on $n$ elements, i.e., the group of the $n$ ! permutations of $n$ elements. To simplify the notation, we shall assume that the empty function $\varnothing: \varnothing \rightarrow \varnothing$ is the (unique) permutation of zero elements. A transposition is a permutation which leaves all the elements fixed except for two adjacent ones, which are transposed, that is 'swapped'. We use $(i+1)$ to denote the transposition of $i$ and $i+1$.

Definition 3.2. (The Category of Permutations) Let $S$ be a set. The category $S y m_{S}^{*}$ has as objects the strings in $S^{\otimes}$, and an arrow $p: u \rightarrow v$ if and only if $p \in \Pi(|u|)$, i.e., $p$ is a permutation of $|u|$ elements, and $v$ is the string obtained by applying the permutation $p$ to $u$, i.e., $v_{p(i)}=u_{i}$.

Arrow composition in $S_{y} m_{S}^{*}$ is obviously given by the product of permutations, i.e., their composition as functions, here denoted by _; _ written in diagrammatic order.

Graphically, we represent an arrow $p: u \rightarrow v$ in $S y m_{S}^{*}$ by drawing a line between $u_{i}$ and $v_{p(i)}$, as for example in Figure 3.

Of course, it is possible to extend the monoidal structure of $S^{\otimes}$ to a tensor product on $S y m_{S}^{*}$, together with natural transformations associated to interchange permutations between strings that make it a symmetric monoidal category (see also Figure 3, where $\gamma$ is the permutation (12)).

Definition 3.3. (Operations on Permutations) Given permutations $p: u \rightarrow v$ and $p^{\prime}: u^{\prime} \rightarrow v^{\prime}$ 


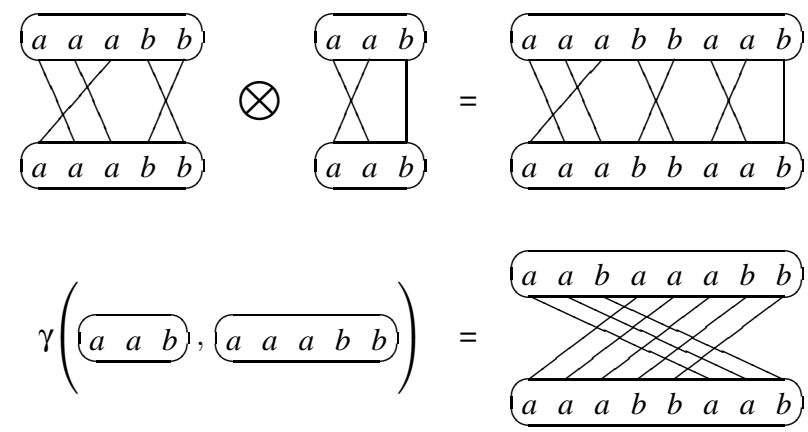

Figure 3. The monoidal structure of $S y m_{S}^{*}$

in $S y m_{S}^{*}$ their parallel composition $p \otimes p^{\prime}: u \otimes u^{\prime} \rightarrow v \otimes v^{\prime}$ is the permutation such that

$$
i \mapsto \begin{cases}p(i) & \text { if } 0<i \leq|u| \\ p^{\prime}(i-|u|)+|u| & \text { if }|u|<i \leq|u|+\left|u^{\prime}\right|\end{cases}
$$

Given $\pi \in \Pi(m)$ and $m$ strings $u_{i} \in S^{\otimes}, i=1, \ldots, m$, the interchange permutation $\pi\left(u_{1}, \ldots, u_{m}\right)$ is the permutation $p$ such that

$$
p(i)=i-\sum_{j=1}^{h-1}\left|u_{j}\right|+\sum_{\pi(j)<\pi(h)}\left|u_{j}\right| \quad \text { if } \sum_{j=1}^{h-1}\left|u_{j}\right|<i \leq \sum_{j=1}^{h}\left|u_{j}\right| .
$$

Clearly, $\otimes$ so defined is associative and furthermore a simple calculation shows that it satisfies the equations

$$
\left(p \otimes p^{\prime}\right) ;\left(q \otimes q^{\prime}\right)=(p ; q) \otimes\left(p^{\prime} ; q^{\prime}\right) \quad \text { and } \quad i d_{u} \otimes i d_{v}=i d_{u \otimes v} .
$$

It follows easily that the mapping $\otimes: S y m_{S}^{*} \times S y m_{S}^{*} \rightarrow S y m_{S}^{*}$ defined by

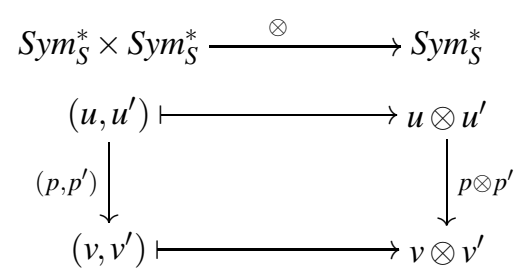

is a functor making $S_{y m}^{*}$ a strict monoidal category. Finally, the symmetric structure of $S y m_{S}^{*}$ is made explicit through the interchange permutations.

Proposition 3.4. (Sym ${ }_{S}^{*}$ is symmetric strict monoidal) For any set $S$, the family of interchange permutations $\gamma=\{\gamma(u, v)\}_{u, v \in S y m_{S}^{*}}$, defined from the permutation $\gamma=(12)$, provides the symmetry isomorphism endowing $\operatorname{Sym}_{S}^{*}$ with a symmetric monoidal structure.

Proof. It is just a matter of performing a few calculations to verify that, for any $p: u \rightarrow u^{\prime}$ and $p^{\prime}: v \rightarrow v^{\prime}$, the equations defining a symmetry isomorphism i.e., equations (6) in Appendix A, which in the current case reduce to

$$
(\gamma(u, v) \otimes w) ;(v \otimes \gamma(u, w))=\gamma(u, v \otimes w)
$$



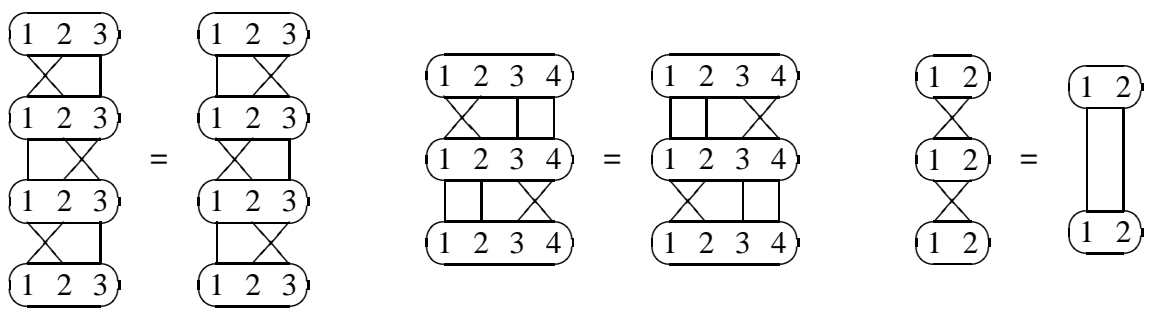

Figure 4. Some instances of the axioms of permutations

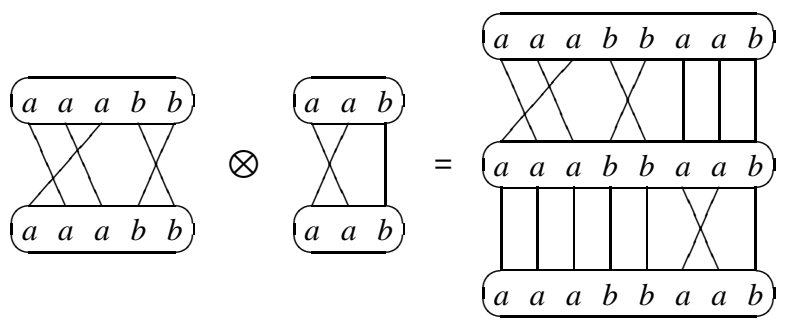

Figure 5. The parallel composition of permutations

$$
\begin{aligned}
\gamma(u, v) ;\left(p^{\prime} \otimes p\right) & =\left(p \otimes p^{\prime}\right) ; \gamma\left(u^{\prime}, v^{\prime}\right) \\
\gamma(u, v) ; \gamma(v, u) & =u \otimes v,
\end{aligned}
$$

hold.

The previous proposition justifies using the name symmetries for the arrows of the groupoid $S y m_{S}^{*}$. As anticipated above, the key point about $S y m_{S}^{*}$ is that it is a free construction. In order to show it, we need the following lemma (Moore 1897; Burnside 1911).

Lemma 3.5. The symmetric group $\Pi(n)$ is (isomorphic to) the group $G$ freely generated from the set of elements $\left\{\tau_{i} \mid 1 \leq i<n\right\}$, modulo the equations (see also Figure 4)

$$
\begin{aligned}
\tau_{i} \tau_{i+1} \tau_{i} & =\tau_{i+1} \tau_{i} \tau_{i+1} \\
\tau_{i} \tau_{j} & =\tau_{j} \tau_{i} \quad \text { if }|i-j| \geq 1 \\
\tau_{i} \tau_{i} & =e ;
\end{aligned}
$$

where $e$ is the neutral element of the group. The equivalence class of $\tau_{i}$ modulo the above equations corresponds to the transposition $(i i+1)$.

We are now ready to show the announced fact about $S y m_{S}^{*}$.

Proposition 3.6. ( $\mathrm{Sym}_{S}^{*}$ is free) Let $S$ be a set, let $\mathrm{C}$ be a symmetric strict monoidal category, and let $f$ be a function from $S$ to the set of objects of $C$. Then, there exists a unique symmetric strict monoidal functor $\mathrm{F}: \operatorname{Sym}_{S}^{*} \rightarrow \mathrm{C}$ extending $f$.

Proof. Let $\otimes$ be the tensor product, $e$ the unit object, and $c: x_{1} \otimes x_{2} \dot{\rightarrow} x_{2} \otimes x_{1}$ the symmetry natural isomorphism of $C$. There is of course a choice forced upon us for the behaviour of $F$ on objects: the monoidal extension of $f$, i.e., the mapping

$$
\mathrm{F}(\varepsilon)=e, \quad \mathrm{~F}(a)=f(a) \text { for } a \in S, \quad \text { and } \quad \mathrm{F}(u \otimes v)=\mathrm{F}(u) \otimes \mathrm{F}(v) \text { for } u, v \in S^{\otimes} .
$$

Concerning morphisms, we know by Lemma 3.5 that each arrow in $S y m_{S}^{*}$ can be written as a 
composition of transpositions. The transposition $(i i+1): u \otimes a \otimes b \otimes v \rightarrow u \otimes b \otimes a \otimes v$, where $u$ is a string of length $i-1$, coincides in $\operatorname{Sym}_{S}^{*}$ with the tensor of $\gamma(a, b): a \otimes b \rightarrow b \otimes a$ with appropriate identities, namely $(u \otimes \gamma(a, b) \otimes v)$. Thus, recalling also that $\varepsilon \otimes \gamma(a, b)=\gamma(a, b)=\gamma(a, b) \otimes \varepsilon$, the following equations define $\mathrm{F}$ on all the arrows of $S y m_{S}^{*}$.

$$
\begin{aligned}
\mathrm{F}(u \otimes \gamma(a, b) \otimes v) & =\mathrm{F}(u) \otimes c_{\mathrm{F}(a), \mathrm{F}(b)} \otimes \mathrm{F}(v) \quad a, b \in S, u, v \in S^{\otimes} \\
\mathrm{F}\left(p ; p^{\prime}\right) & =\mathrm{F}\left(p^{\prime}\right) \circ \mathrm{F}(p) .
\end{aligned}
$$

Observe that both the equations (4) are forced by the definition of symmetric strict monoidal functor (see axioms (7) in Appendix A). It follows that the extension of $f$ to a strict monoidal functor, if it exists, is unique and must be given by (4). Then, in order to conclude the proof, we only need to show that $\mathrm{F}$ is well defined and that it is a symmetric monoidal functor.

For the former, it is enough to show that the axioms (3) of Lemma 3.5 are preserved by $F$. Concerning axioms (3), the third one matches directly with the fact that the inverse of $c_{\mathrm{F}(a), \mathrm{F}(b)}$ is $c_{\mathrm{F}(b), \mathrm{F}(a)}$, while the second one follows easily from the fact that $\otimes$ is a functor. In fact, in the hypothesis, we have $\tau_{i}=(u \otimes \gamma(a, b) \otimes v \otimes c \otimes d \otimes w)$ and $\tau_{j}=(u \otimes b \otimes a \otimes v \otimes \gamma(c, d) \otimes w)$. Thus, we have that

$$
\begin{aligned}
& \mathrm{F}\left(\tau_{i} ; \tau_{j}\right)=\left(\mathrm{F}(u) \otimes \mathrm{F}(b) \otimes \mathrm{F}(a) \otimes \mathrm{F}(v) \otimes c_{\mathrm{F}(c), \mathrm{F}(d)} \otimes \mathrm{F}(w)\right) \circ \\
&\left(\mathrm{F}(u) \otimes c_{\mathrm{F}(a), \mathrm{F}(b)} \otimes \mathrm{F}(v) \otimes \mathrm{F}(c) \otimes \mathrm{F}(d) \otimes \mathrm{F}(w)\right) \\
&=\left(\mathrm{F}(u) \otimes c_{\mathrm{F}(a), \mathrm{F}(b)} \otimes \mathrm{F}(v) \otimes c_{\mathrm{F}(c), \mathrm{F}(d)} \otimes \mathrm{F}(w)\right) \\
&=\left(\mathrm{F}(u) \otimes c_{\mathrm{F}(a), \mathrm{F}(b)} \otimes \mathrm{F}(v) \otimes \mathrm{F}(d) \otimes \mathrm{F}(c) \otimes \mathrm{F}(w)\right) \circ \\
&\left(\mathrm{F}(u) \otimes \mathrm{F}(a) \otimes \mathrm{F}(b) \otimes \mathrm{F}(v) \otimes c_{\mathrm{F}(c), \mathrm{F}(d)} \otimes \mathrm{F}(w)\right) \\
&=
\end{aligned}
$$

Finally, exploiting the naturality and the coherence equations for $c$, a similar calculation shows that $\mathrm{F}\left(\tau_{i} ; \tau_{i+1} ; \tau_{i}\right)=\mathrm{F}\left(\tau_{i+1} ; \tau_{i} ; \tau_{i+1}\right)$.

Let us prove that $\mathrm{F}$ is a symmetric monoidal functor. Since $\mathrm{C}$ is a symmetric strict monoidal category, we have $c_{e, x}=c_{e \otimes e, x}=c_{e, x} \otimes e \circ e \otimes c_{e, x}=c_{e, x} \circ c_{e, x}$, and since $c_{e, x}$ is invertible, it follows that $c_{e, x}=i d_{x}$. Of course, the same holds for any other symmetric strict monoidal category. Therefore, since $\mathrm{F}\left(i d_{u}\right)=\mathrm{F}(\gamma(\varepsilon, u))$ and $c_{e, \mathrm{~F}(u)}=i d_{\mathrm{F}(u)}$, we have that $\mathrm{F}\left(i d_{u}\right)=i d_{\mathrm{F}(u)}$. This, together with the second of the equations (4), means that $\mathrm{F}$ is a functor.

Observe further that, for $p: u \rightarrow v$ and $p^{\prime}: u^{\prime} \rightarrow v^{\prime}$ in $S y m_{S}^{*}$, we have $p \otimes p^{\prime}=\left(p \otimes u^{\prime}\right) ;\left(v \otimes p^{\prime}\right)$ (see also Figure 5). Then, by definition,

$$
\mathrm{F}\left(p \otimes p^{\prime}\right)=\mathrm{F}\left(v \otimes p^{\prime}\right) \circ \mathrm{F}\left(p \otimes u^{\prime}\right)=\left(\mathrm{F}(v) \otimes \mathrm{F}\left(p^{\prime}\right)\right) \circ\left(\mathrm{F}(p) \otimes \mathrm{F}\left(u^{\prime}\right)\right)=\mathrm{F}(p) \otimes \mathrm{F}\left(p^{\prime}\right),
$$

i.e., $\mathrm{F}$ is a strict monoidal functor.

Finally, thanks to the first of the coherence axioms for symmetries, $\gamma(a, b \otimes c)=(\gamma(a, b) \otimes c)$; $(b \otimes \gamma(a, c))$ and thus, by the aforesaid axiom and by the corresponding coherence axiom of $c$,

$$
\begin{aligned}
\mathrm{F}(\gamma(a, b \otimes c)) & =\mathrm{F}((\gamma(a, b) \otimes c) ;(b \otimes \gamma(a, c))) \\
& =\left(\mathrm{F}(b) \otimes c_{\mathrm{F}(a), \mathrm{F}(c)}\right) \circ\left(c_{\mathrm{F}(a), \mathrm{F}(b)} \otimes \mathrm{F}(c)\right) \\
& =c_{\mathrm{F}(a), \mathrm{F}(b) \otimes \mathrm{F}(c)}=c_{\mathrm{F}(a), \mathrm{F}(b \otimes c)} .
\end{aligned}
$$


Now, by considering the inverses of the arrows appearing in the first coherence axiom, we have $\gamma(a \otimes b, c)=(a \otimes \gamma(b, c)) ;(\gamma(a, c) \otimes b)$ and $c_{\mathrm{F}(a \otimes b), \mathrm{F}(c)}=\left(c_{\mathrm{F}(a), \mathrm{F}(c)} \otimes \mathrm{F}(b)\right) \circ\left(\mathrm{F}(a) \otimes c_{\mathrm{F}(b), \mathrm{F}(c)}\right)$. Therefore, it follows easily by induction that $\mathrm{F}(\gamma(u, v))=c_{\mathrm{F}(u), \mathrm{F}(v)}$. Then, $\mathrm{F}$ maps each component of the symmetry natural isomorphism of $\operatorname{Sym}_{S}^{*}$ to the corresponding component of $c$, and, therefore, we conclude that $\mathrm{F}$ is a symmetric monoidal functor.

This result proves that the mapping $S \mapsto S y m_{S}^{*}$ extends to a left adjoint functor from Set to SSMC, the standard category of symmetric strict monoidal (small) categories and symmetric strict monoidal functors, whose right adjoint send each such category to its set of objects. Equivalently, we can say that $S y m_{S}^{*}$ is, up to isomorphism, the free symmetric strict monoidal category on the set $S$. This is remarked by the following corollary, which also proves that $S_{y m}^{*}$, the category of symmetries of $Q(N)$, is isomorphic to $S y m_{S_{N}}^{*}$.

Corollary 3.7. ( $S y m_{S}^{*}$ is free) Let $S$ be the category whose monoid of objects is $S^{\otimes}$, the free monoid on $S$, and whose arrows are freely generated from the family of arrows $i d_{u}: u \rightarrow u$ and $c_{u, v}: u \otimes v \rightarrow v \otimes u$, for $u, v \in S^{\otimes}$, subject to axioms (1) and (2). Then $S$ and $S y m_{S}^{*}$ are isomorphic.

It follows that $Q(N)$ can be described as the symmetric strict monoidal category built over the subcategory of symmetries $S y m_{S_{N}}^{*}$ by adding freely the arrows given by

$$
\frac{t: \mathcal{M}(u) \rightarrow \mathcal{M}(v) \text { in } T_{N}}{t_{u, v}: u \rightarrow v \text { in } Q(N)}
$$

and quotienting modulo axiom $(\Phi)$. In force of these facts, in the following we shall safely confuse $\operatorname{Sym}_{N}^{*}$ and $S y m_{S_{N}}^{*}$ and, therefore, the symmetry $c_{u, v} \in S y m_{N}^{*}$ and the corresponding permutation $\gamma(u, v) \in \operatorname{Sym}_{S_{N}}^{*}$.

We show next that $Q($ (_) can be lifted to a functor from the category of Petri nets to an appropriate category of symmetric strict monoidal categories and equivalence classes of symmetric strict monoidal functors. The role of such an equivalence is to take into account that we look at the strings of $S_{N}^{\otimes}$ as concrete representatives of the multisets of $S_{N}^{\oplus}$ and, therefore, we want to consider as perfectly equal those functors which differ only by picking up different, yet compatible, linearizations of multisets. The task is not very difficult now, since most of the work has been done in the proof of Proposition 3.6.

We start by showing that $Q(-)$ is a pseudo-functor from Petri to SSMC in the sense made explicit by Proposition 3.8 below. More precisely, we extend $Q(-)$ to a mapping from Petri net morphisms to symmetric strict monoidal functors in such a way that identities are preserved strictly, while net morphism composition is preserved only up to a monoidal natural isomorphism (see Appendix A). In order to do that, the key point which is still missing is to be able to 'lift' morphisms of free commutative monoids to morphisms of free non-commutative monoids. To achieve this, we proceed as follows.

Let FMon and FCMon be, respectively, the category of free and free commutative monoids, together with their homomorphisms. Consider the obvious quotient functor $\left({ }_{-}\right)^{b}$ : FMon $\rightarrow$ FCMon. Explicitly, $\left(S^{\otimes}\right)^{b}=S^{\otimes} /\{x y=y x\}=S^{\oplus}$, whilst the action of $\left({ }_{-}\right)^{b}$ on $f: S_{0}^{\otimes} \rightarrow S_{1}^{\otimes}$ gives the unique homomorphism $f^{b}: S_{0}^{\oplus} \rightarrow S_{1}^{\oplus}$ such that $f^{b}(a)=\mathcal{M}(f(a))$ for all $a \in S$. It is easy to verify that, when considered as a morphism of reflexive graphs, $\left({ }_{-}\right)^{b}$ admits a section, i.e., a reflexive graph morphism $\left({ }_{-}\right)^{\natural}$ : FCMon $\rightarrow$ FMon such that $\left((-)^{\natural}\right)^{b}=i d_{\text {FCMon }}$. In other words, $\left({ }_{-}\right)^{\natural}$ maps $S^{\oplus}$ to $S^{\otimes}$ and assigns a 'linearization' $f^{\natural}: S_{0}^{\otimes} \rightarrow S_{1}^{\otimes}$ to $f: S_{0}^{\oplus} \rightarrow S_{1}^{\oplus}$ in such a way that 
- $\mathcal{M}\left(f^{\natural}(a)\right)=f(a)$, for all $f: S_{0}^{\oplus} \rightarrow S_{1}^{\oplus}$ and all $a \in S_{0} ;$

- $\left(i d_{S^{\oplus}}\right)^{\natural}=i d_{S^{\otimes}}$, for all sets $S$.

Fixed one such (-) $)^{\natural}$, we can define the action of $Q(-)$ on Petri net morphisms. Observe that, in principle, choosing two different different for $\left({ }_{-}\right)^{b}$ gives two different - yet equivalent extensions of $Q($ (_) to a pseudo-functor. We would like to remark that this apparent arbitrariness of $Q(-)$ is not at all a concern, since the relevant fact we want to show now is that such an extension exists. Moreover, we shall see shortly that introducing the category QSSMC one can completely dispense with $\left({ }_{-}\right)^{\natural}$, i.e., the 'choice mapping' $\left({ }^{\natural}\right)^{\natural}$ is actually, in a precise mathematical sense, irrelevant.

Proposition 3.8. ( $Q$ (_): Petri $\rightarrow$ SSMC) Let $f: N_{0} \rightarrow N_{1}$ be a morphism in Petri. Then, there exists a symmetric strict monoidal functor $Q(f): Q\left(N_{0}\right) \rightarrow Q\left(N_{1}\right)$ which extends $f$. Moreover, $Q\left(i d_{N}\right)=i d_{Q(N)}$ and $Q(g \circ f) \dot{\rightarrow}(g) \circ Q(f)$ via a monoidal natural isomorphism (see Appendix A) whose components are all symmetries.

Proof. Since $f_{S}$ is a monoid homomorphism from $S_{N_{0}}^{\oplus}$ to $S_{N_{1}}^{\oplus}$, we consider the homomorphism $f_{S}^{\natural}: S_{N_{0}}^{\otimes} \rightarrow S_{N_{1}}^{\otimes}$. By the freeness of $S y m_{N_{0}}^{*}$, such a morphism can be extended (uniquely) to a symmetric strict monoidal functor $\mathrm{F}_{S}: \operatorname{Sym}_{N_{0}}^{*} \rightarrow Q\left(N_{1}\right)$ and, thus, to a functor F: $Q\left(N_{0}\right) \rightarrow Q\left(N_{1}\right)$, defined as the unique symmetric strict monoidal functor which coincides with $\mathrm{F}_{S}$ on $\operatorname{Sym}_{N_{0}}$ and maps $t_{u, v}: u \rightarrow v$ to $f_{T}(t)_{\mathrm{F}(u), \mathrm{F}(v)}: \mathrm{F}(u) \rightarrow \mathrm{F}(v)$. Since monoidal functors map symmetries to symmetries, and since $f_{T}(t)$ is a transition of $N_{1}$, it follows immediately that $\mathrm{F}$ respects axiom $(\Phi)$, i.e., that $\mathrm{F}$ is well defined.

We show next that the above definition makes $Q(-)$ into a pseudo-functor. First of all, since $Q(f)$ is uniquely determined by $f_{T}$ and $f_{S}^{\natural}$, by the property $\left(i d_{S^{\oplus}}\right)^{\natural}=i d_{S^{\otimes}}$ of $(-)^{\natural}$, it follows that $Q\left(i d_{N}\right): Q(N) \rightarrow Q(N)$ is the identity functor. Now, let $f: N_{0} \rightarrow N_{1}$ and $g: N_{1} \rightarrow N_{2}$ be morphisms of nets. Observe that all $a \in S_{N_{0}}$, the string $\left(g_{S} \circ f_{S}\right)^{\natural}(a)$ is a permutation of $g_{S}^{\natural}\left(f_{S}^{\natural}(a)\right)$ and that, therefore, there exists a symmetry $s_{a}: \mathcal{Q}(g \circ f)(a) \rightarrow \mathcal{Q}(g) \circ Q(f)(a)$ in $\mathcal{Q}\left(N_{2}\right)$. Then, for $u=u_{1} \cdots u_{n} \in S_{N_{0}}^{\otimes}$, take $s_{u}$ to be $s_{u_{1}} \otimes \cdots \otimes s_{u_{n}}: \mathcal{Q}(g \circ f)(u) \rightarrow Q(g) \circ Q(f)(u)$. We claim that the family $\left\{s_{u} \mid u \in S_{N_{0}}^{\otimes}\right\}$ is a natural transformation $Q(g \circ f) \doteq Q(g) \circ Q(f)$. Since $s$ is clearly monoidal and each $s_{u}$ is a symmetry isomorphism, this concludes the proof.

We proceed by induction on the structure of $\alpha$ to show that, for any $\alpha: u \rightarrow v$ in $Q\left(N_{0}\right)$, we have

$$
Q(g \circ f)(\alpha) ; s_{v}=s_{u} ; Q(g) \circ Q(f)(\alpha) .
$$

The key to the proof is that $s$ is monoidal, i.e., $s_{u \otimes v}=s_{u} \otimes s_{v}$, as a simple inspection of the definition shows. If $\alpha$ is an identity, then the claim is obvious. Moreover, if $\alpha$ is a transition $t_{u, v}$, then $Q(g \circ f)(\alpha)$ and $Q(g) \circ Q(f)(\alpha)$ are instances of the same transition of $N_{2}$, and the thesis follows immediately from axiom $(\Phi)$. Suppose now that $\alpha=c_{u, v}$, a symmetry of $Q\left(N_{0}\right)$. Since $Q(g \circ f)$ and $Q(g) \circ Q(f)$ are symmetric strict monoidal functors, the equation we have to prove reduces to

$$
c_{Q(g \circ f)(u), Q(g \circ f)(v)} ; s_{v} \otimes s_{u}=s_{u} \otimes s_{v} ; c_{Q(g) \circ Q(f)(u), Q(g) \circ Q(f)(v)},
$$

which certainly holds since $\left\{c_{u, v} \mid u, v \in S_{N_{0}}^{\otimes}\right\}$ is a natural transformation $x_{1} \otimes x_{2} \rightarrow x_{2} \otimes x_{1}$. If $\alpha=\alpha_{0} \otimes \alpha_{1}$, with $\alpha_{i}: u_{i} \rightarrow v_{i}$, then, for $i=0,1$, it follows from the induction hypothesis that $Q(g \circ f)\left(\alpha_{i}\right) ; s_{v_{i}}=s_{u_{i}} ; Q(g) \circ Q(f)\left(\alpha_{i}\right)$, whence

$$
Q(g \circ f)\left(\alpha_{0}\right) \otimes Q(g \circ f)\left(\alpha_{1}\right) ; s_{v_{0} \otimes v_{1}}=s_{u_{0} \otimes u_{1}} ; Q(g) \circ Q(f)\left(\alpha_{0}\right) \otimes Q(g) \circ Q(f)\left(\alpha_{1}\right),
$$


which is $Q(g \circ f)(\alpha) ; s_{v}=s_{u} ; Q(g) \circ Q(f)(\alpha)$. Finally, in case $\alpha=\alpha_{0} ; \alpha_{1}$, with $\alpha_{0}: u \rightarrow w$ and $\alpha_{1}: w \rightarrow v$, the induction is maintained by pasting the two commutative squares in the following diagram, which exists by the induction hypothesis

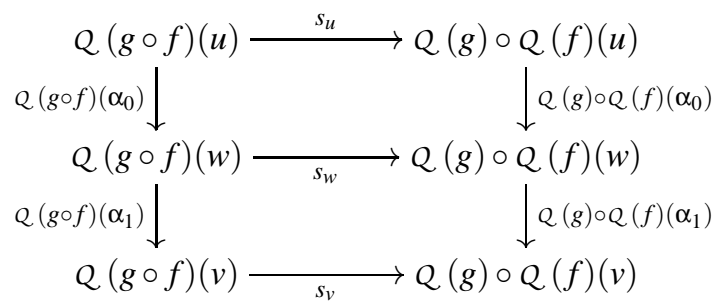

Thus, $Q(g \circ f)(\alpha) ; s_{v}=s_{u} ; Q(g) \circ Q(f)(\alpha)$, which concludes the proof.

Therefore, due to technical reasons depending exclusively on the lack of functoriality of $\left({ }_{-}\right)^{\natural}$, $Q$ (-) fails to be a functor from Petri to SSMC. It is only a pseudo-functor. However, it is worth remarking that this failure is intrinsically different from the situation for $\mathcal{P}(-)$, and that the pseudofunctoriality of $Q(-)$ is already a valuable result. In fact, in the case of $P(-)$, we cannot lift net morphisms to functors between the categories of processes, a failure which may possibly rise doubts on the structure chosen to represent the processes of the single net, while in the case of $Q(-)$, we just cannot define composition of the lifted morphisms better than 'up to isomorphism'. Of course, this depends on the fact that our idea of 'strings as representatives of multisets' is not reflected adequately in SSMC, and simply brings us to the conclusion that SSMC is not quite the correct target category for the functorial construction we are looking for. Indeed, as we shall see in the following, it is easy to identify a category QSSMC of symmetric strict monoidal categories such that $Q\left({ }_{-}\right)$is a functor Petri $\rightarrow$ QSSMC. Actually, this construction is already implicit in Proposition 3.8 and corresponds to taking an appropriate quotient of SSMC.

Definition 3.9. (Symmetric Petri Categories) A symmetric Petri category is a symmetric strict monoidal category C in SSMC whose monoid of objects is the free monoid $S^{\otimes}$ for some set $S$.

For any pair $C$ and $D$ of symmetric Petri categories, consider the binary relation $R C, D$ on the symmetric strict monoidal functors from $C$ to $D$ defined by $F \mathcal{R} C, D G$ if and only if there exists a monoidal natural isomorphism $\sigma: \mathrm{F} \rightarrow \mathrm{G}$ whose components are all symmetries. Clearly, $\mathcal{R} \mathrm{C}, \mathrm{D}$ is an equivalence relation. Moreover, if $\mathrm{H}: \mathrm{C}^{\prime} \rightarrow \mathrm{C}$ and $\mathrm{K}: \mathrm{D} \rightarrow \mathrm{D}^{\prime}$ are symmetric strict monoidal

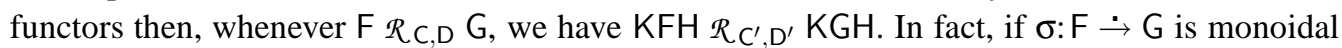
and all its components are symmetries, then so is $\mathrm{K} \sigma \mathrm{H}: \mathrm{KFH} \rightarrow \mathrm{KGH}$. In other words, the family of equivalences $\mathcal{R}$ is actually a congruence with respect to functor composition. Therefore, the following definition makes sense.

Definition 3.10. (The category QSSMC) Let QSSMC be the quotient of the full subcategory of SSMC consisting of the symmetric Petri categories modulo the congruence $R$.

Of course, concerning QSSMC there is the following easy result.

Proposition 3.11. ( $Q$ (_): Petri $\rightarrow$ QSSMC) $Q$ (_) extends to a functor from Petri to QSSMC.

Proof. For $f: N_{0} \rightarrow N_{1}$, define $Q(f)$ to be the equivalence class of the functor in SSMC from $Q\left(N_{0}\right)$ to $Q\left(N_{1}\right)$ described in Proposition 3.8. It follows immediately from the proof of that proposition that $Q(-)$ is a functor from Petri to QSSMC. 
Observe that, when describing $Q(f)$ in QSSMC, there is no need to consider the section $\left(_{-}\right)^{\natural}$ of $\left({ }_{-}\right)^{b}$, since the extensions of $f$ to a symmetric strict monoidal functor corresponding to different sections or $\left({ }_{-}\right)^{b}$ yield the same functor in QSSMC. In fact, if $\left(_{-}\right)^{\natural}$ and $\left(_{-}\right)^{\sharp}$ are sections of $\left(_{-}\right)^{b}$, for any morphism $f: N_{0} \rightarrow N_{1}$ and all $a \in S_{N_{0}}$, the string $f^{\natural}(a)$ is a permutation of $f^{\sharp}(a)$. Then, we can proceed as in the proof of Proposition 3.8 to choose a monoidal natural isomorphism composed only of symmetries such that $Q^{\natural}(f) \stackrel{\rightarrow}{\sharp}(f)$.

However, the category QSSMC is still too general for our purposes. In particular, it is easily noticed that $Q(-)$ is not full (though it is faithful), i.e., that there are functors from $Q\left(N_{0}\right)$ to $Q\left(N_{1}\right)$ in QSSMC which do not correspond to any morphism from $N_{0}$ to $N_{1}$ in Petri. This means that QSSMC has too little structure to represent net behaviours with enough precision; equivalently, since the structure of the objects of a category $C$ is 'encoded' in the morphisms of $C$, it means that the morphisms of QSSMC do not capture the structure of symmetric Petri categories precisely enough. Specifically, the transitions, which are definitely primary components of nets, and as such are treated by the morphisms in Petri, have no corresponding notion in QSSMC: we need to identify such a notion and refine the choice of the category of net computations accordingly.

Notation. Given a symmetric monoidal category C, let $\operatorname{Sym}_{\mathrm{C}}$ indicate the subcategory of C consisting of the symmetries, i.e., of those arrows which are built up from identities and components of symmetry isomorphism of $\mathrm{C}$.

The key to accomplishing our task is the following observation about axiom $(\Phi)$ in Definition 3.1: as already mentioned, it simply expresses that the collection of arrows $t_{u, v}$ of $Q(N)$, for $t \in T_{N}$ and $u, v \in S_{N}^{\otimes}$, is a natural transformation. Namely, for C a symmetric Petri category with objects $S^{\otimes}$, and $v$ a multiset in $S^{\oplus}$, let $S y m_{C, v}$ be the subcategory of C consisting of those objects $u \in S^{\otimes}$ such that $\mathcal{M}(u)=v$ and the symmetries between them, and let $i_{\mathrm{C}, v}$ be the inclusion of $\operatorname{Sym}_{\mathrm{C}, v}$ in C. Then, for $v, v^{\prime} \in S^{\oplus}$, one obtains a pair of parallel functors $\pi_{\mathrm{C}, v, v^{\prime}}^{0}$ and $\pi_{\mathrm{C}, v, v^{\prime}}^{1}$ by composing $i n_{\mathrm{C}, v}$ and $i n_{\mathrm{C}, v^{\prime}}$ respectively with the first and with the second projection of $\operatorname{Sym}_{\mathrm{C}, \mathrm{v}} \times \operatorname{Sym}_{\mathrm{C}, \mathrm{v}^{\prime}}$.

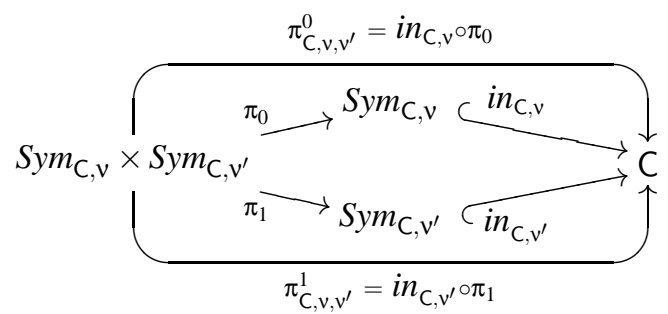

It follows directly from the definitions that, when $C$ is $Q(N)$, axiom $(\Phi)$ states exactly that, for all $t: v \rightarrow v^{\prime} \in T_{N}$, the set $\left\{t_{u, v} \mid \mathcal{M}(u)=v, \mathcal{M}(v)=v^{\prime}\right\}$ is a natural transformation from $\pi_{Q(N), v, v^{\prime}}^{0}$ to $\pi_{Q(N), v, v^{\prime}}^{1}$.

A further very relevant property of the transitions of $N$ when considered as arrows of $Q(N)$ is that of being decomposable as a tensor only trivially and as a composition only by means of symmetries. This is easily captured by the following notion of primitive arrow.

Definition 3.12. (Primitive Arrows) Let $C$ be a symmetric Petri category. An arrow $\tau$ in $C$ is primitive if 
(i) $\tau$ is not a symmetry;

(ii) $\tau=\alpha ; \beta \quad$ implies $\alpha$ is a symmetry and $\beta$ is primitive, or vice versa;

(iii) $\tau=\alpha \otimes \beta$ implies $\quad \alpha=i d_{\varepsilon}$ and $\beta$ is primitive, or vice versa.

A simple inspection of Definition 3.1 shows that the only primitive arrows in $Q(N)$ are the arrows $t_{u, v}$, for $t: \mathcal{M}(u) \rightarrow \mathcal{M}(v)$ a transition of $N$. As a consequence, the natural transformations $\tau: \pi_{Q(N), v, v^{\prime}}^{0} \dot{\rightarrow} \pi_{Q(N), v, v^{\prime}}^{1}$ whose components are primitive are in one-to-one correspondence with the transitions of $N$. Following the usual categorical paradigm, we then use the properties that characterize the transitions of $N$ in $Q(N)$, expressed in abstract categorical terms, to define the notion of transition in any symmetric Petri category.

Definition 3.13. (Transitions in Symmetric Petri Categories) Let $C$ be a symmetric Petri category and let $S^{\otimes}$ be its monoid of objects. A transition of C is a natural transformation $\tau: \pi_{C, v, v}^{0} \dot{\rightarrow} \pi_{C, v, v^{\prime}}^{1}$, for $v, v^{\prime}$ in $S^{\oplus}$, whose components $\tau_{u, v}$ are primitive arrows of $C$.

It is now clear what the extra structure required in QSSMC is: transitions must be preserved by morphisms of symmetric Petri categories. Formally, for C and D in QSSMC and F: C $\rightarrow D$ in SSMC, $\mathrm{F}$ respects transitions if, for each transition $\tau: \pi_{C, v, v^{\prime}}^{0} \dot{\rightarrow} \pi_{C, v, v^{\prime}}^{1}$ of $C$, there exists a transition $\tau^{\prime}: \pi_{\mathrm{D}, \bar{v}, \bar{v}^{\prime}}^{0} \dot{\rightarrow} \pi_{\mathrm{D}, \bar{v}, \bar{v}^{\prime}}^{1}$ of D such that $\mathrm{F}\left(\tau_{u, v}\right)=\tau_{\mathrm{F}(u), \mathrm{F}(v)}^{\prime}$ for all $(u, v)$ in $\operatorname{Sym}_{\mathrm{C}, v} \times \operatorname{Sym}_{\mathrm{C}, v^{\prime}}$; in this case, we say that $\tau^{\prime}$ corresponds to $\tau$ via $\mathrm{F}$.

Lemma 3.14. If $\mathrm{F}: \mathrm{C} \rightarrow \mathrm{D}$ respects transitions, then for any transition $\tau$ of $\mathrm{C}$, there exists a unique transition $\tau^{\prime}$ of $D$ which corresponds to $\tau$ via $F$.

Proof. First observe that, for any symmetric Petri category $C$ and any pair of natural transformations $\tau, \tau^{\prime}: \pi_{\mathrm{C}, v, v}^{0} \dot{\rightarrow} \pi_{\mathrm{C}, v, v^{\prime}}^{1}$ whenever $\tau_{u, v}=\tau_{u, v}^{\prime}$ for some $u$ and $v$, then $\tau=\tau^{\prime}$. In fact, for any $u^{\prime}$ and $v^{\prime}$ there exists $\left(s, s^{\prime}\right):\left(u^{\prime}, v\right) \rightarrow\left(u, v^{\prime}\right)$ in $\operatorname{Sym}_{\mathrm{C}, v} \times \operatorname{Sym}_{\mathrm{C}, v^{\prime}}$, and then $\tau_{u^{\prime}, v^{\prime}}=s ; \tau_{u, v} ; s^{\prime}=$ $s ; \tau_{u, v}^{\prime} ; s^{\prime}=\tau_{u^{\prime}, v^{\prime}}^{\prime}$

Now consider the transitions $\tau^{\prime}$ and $\tau^{\prime \prime}$ of $\mathrm{D}$ and suppose that they both correspond to $\tau$ via $\mathrm{F}$. Then, $\mathrm{F}\left(\tau_{u, v}\right)=\tau_{\mathrm{F}(u), \mathrm{F}(v)}^{\prime}=\tau_{\mathrm{F}(u), \mathrm{F}(v)}^{\prime \prime}$, which implies $\tau^{\prime}=\tau^{\prime \prime}$.

The previous lemma shows that any symmetric strict monoidal functor which preserves transitions defines a mapping between the respective sets of transitions. The next lemma proves that this property extends to the arrows of QSSMC. It follows then immediately that Definition 3.16 is well given.

Lemma 3.15. If $F \mathcal{R} G$, then $F$ respects transitions if and and only if $G$ does so, and then $\tau^{\prime}$ corresponds to $\tau$ via $F$ if and only if $\tau^{\prime}$ corresponds to $\tau$ via $G$.

Proof. Let $\sigma: \mathrm{F} \rightarrow \mathrm{G}: \mathrm{C} \rightarrow \mathrm{D}$ be a monoidal natural isomorphism whose components are symmetries, suppose that $F$ respects transitions, and consider a transition $\tau: \pi_{\mathrm{C}, v, v^{\prime}}^{0} \dot{\rightarrow} \pi_{\mathrm{C}, v, v^{\prime}}^{1}$. By hypothesis, there exists a transition $\tau^{\prime}: \pi_{\mathrm{D}, \bar{v}, \bar{v}^{\prime}}^{0} \dot{\rightarrow} \pi_{\mathrm{D}, \bar{v}, \bar{v}^{\prime}}^{1}$ of $\mathrm{D}$ such that $\mathrm{F}\left(\tau_{u, v}\right)=\tau_{\mathrm{F}(u), \mathrm{F}(v)}^{\prime}$ for all $(u, v) \in \operatorname{Sym}_{\mathrm{C}, v} \times \operatorname{Sym}_{\mathrm{C}, v^{\prime}}$. Then, by naturality of $\sigma, \mathrm{G}\left(\tau_{u, v}\right)=\sigma_{u}^{-1} ; \tau_{\mathrm{F}(u), \mathrm{F}(v)}^{\prime} ; \sigma_{v}$, and therefore, by naturality of $\tau^{\prime}, \mathrm{G}\left(\tau_{u, v}\right)=\tau_{\mathrm{G}(u), \mathrm{G}(v)}^{\prime}$, and the proof is concluded.

Definition 3.16. (Symmetric Petri Morphisms and the Category SPetriCat) A morphism of symmetric Petri categories is an arrow in QSSMC which respects transitions. We shall use SPetriCat denote the subcategory of QSSMC consisting of the symmetric Petri categories and their morphisms.

Finally, it is easy to prove that $Q(-)$ is actually a functor to SPetriCat. 
Proposition 3.17. ( $Q$ (_): Petri $\rightarrow$ SPetriCat) The functor $Q\left({ }_{-}\right)$restricts to a functor from Petri to SPetriCat.

Proof. It is enough to verify that, for any morphism $f: N_{0} \rightarrow N_{1}$ in Petri, a representative $\mathrm{F}$ of $Q(f)$ respects transitions. But this follows at once, since $f_{T}$ is a function from $T_{N_{0}}$ to $T_{N_{1}}$, since $\mathrm{F}\left(t_{u, v}\right)=f_{T}(t)_{\mathrm{F}(u), \mathrm{F}(v)}$, and since the transitions of $Q\left(N_{i}\right)$ are exactly the natural transformations $\left\{t_{u, v} \mid \mathcal{M}(u)=v, \mathcal{M}(v)=v^{\prime}\right\}$, for $t: v \rightarrow v^{\prime} \in T_{N_{i}}$.

Interestingly enough, we can identify a functor from SPetriCat to Petri which is a coreflection right adjoint to $Q(-)$. It is worth remarking that this answers a possible legitimate doubt about the category SPetriCat: in principle, in fact, the functoriality of $Q(-)$ could be due to a very tight choice of the target category, e.g., the congruence $R$ could induce too many isomorphisms of categories and then $Q(-)$ could make undesirable identifications of nets. The existence of a coreflection right adjoint to $Q(-)$ is, of course, the best possible proof of the adequacy of SPetriCat: it implies that Petri is embedded in it fully and faithfully. More precisely, Petri is (equivalent to) a coreflective subcategory of SPetriCat. This result supports our claim that SPetriCat is an axiomatization of the category of net computations.

Proposition 3.18. $\left(Q\left({ }_{-}\right) \dashv \mathcal{X}\left(\_\right):\right.$Petri $\rightarrow$ SPetriCat) Let $C$ be a symmetric Petri category, and let $S^{\otimes}$ be its monoid of objects. Define $\mathcal{N}(\mathrm{C})$ to be the Petri net $\left(\partial^{0}, \partial^{1}: T \rightarrow S^{\oplus}\right)$, where

- $T$ is the set of transitions $\tau: \pi_{\mathrm{C}, v, v^{\prime}}^{0} \dot{\rightarrow} \pi_{\mathrm{C}, v, v^{\prime}}^{1}$ of $\mathrm{C}$;

$-\partial^{0}\left(\tau: \pi_{\mathrm{C}, v, v^{\prime}}^{0} \dot{\rightarrow} \pi_{\mathrm{C}, v, v^{\prime}}^{1}\right)=\mathrm{v}$

$-\partial^{1}\left(\tau: \pi_{C, v, v^{\prime}}^{0} \dot{\rightarrow} \pi_{C, v, v^{\prime}}^{1}\right)=v^{\prime}$.

Then, $\mathcal{N}(-)$ extends to a functor SPetriCat $\rightarrow$ Petri which is right adjoint to $Q(-)$. In addition, since the unit is an isomorphism, the adjunction is a coreflection.

Proof. Given a symmetric Petri category C, there is a (unique) symmetric strict monoidal functor $\varepsilon_{\mathrm{C}}: \mathcal{Q} \mathcal{N}(C) \rightarrow C$ which is the identity on the objects and which sends the component at $(u, v)$ of the transition $\tau: v \rightarrow v^{\prime}$ of $\mathcal{N}(\mathrm{C})$, in the following denoted by $[\tau]_{u, v}$, to the component $\tau_{u, v}$ of the corresponding natural transformation $\tau: \pi_{\mathrm{C}, v, v^{\prime}}^{0} \rightarrow \pi_{\mathrm{C}, v, v^{\prime}}^{1}: \operatorname{Sym}_{\mathrm{C}, v} \times \operatorname{Sym}_{\mathrm{C}, v^{\prime}} \rightarrow \mathrm{C}$. In fact, by naturality of $\tau$, we have that $s ; \tau_{u^{\prime}, v^{\prime}}=\tau_{u, v} ; s^{\prime}$ for any symmetries $s: u \rightarrow u^{\prime}$ and $s^{\prime}: v \rightarrow v^{\prime}$ in $\operatorname{Sym}_{\mathrm{C}}$. It follows then directly from Definition 3.1 that the conditions above define $\varepsilon_{\mathrm{C}}$ (uniquely) as a symmetric strict monoidal functor from $Q \mathcal{N}(\mathrm{C})$ to $\mathrm{C}$. In addition, since it clearly preserves transitions, we have that $\varepsilon_{C}$ is a (representative of a) morphism of symmetric Petri categories. We shall prove that $\varepsilon_{\mathrm{C}}$ enjoys the following couniversal property: for each $\mathrm{K}: Q(N) \rightarrow \mathrm{C}$ in SPetriCat, there exists a unique morphism $f: N \rightarrow \mathcal{N}(\mathrm{C})$ in Petri such that the following diagram commutes.

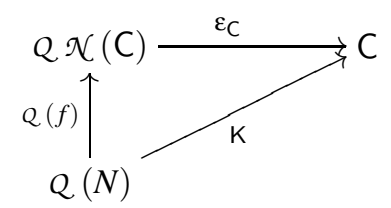

This proves that $\mathcal{N}\left({ }_{-}\right)$is right adjoint to $\mathcal{Q}(-)$, in symbols, $Q\left({ }_{-}\right) \dashv \mathcal{N}\left({ }_{-}\right)$.

Let $S^{\otimes}$ denote the monoid of objects of C, let $\left(\partial^{0}, \partial^{1}: T \rightarrow S^{\oplus}\right)$ be $\mathcal{N}(\mathrm{C})$, and let $\mathrm{F}$ be any representative of K. Since the object component of $F$ is a monoid homomorphism, we have $\mathcal{M}(\mathrm{F}(u))=\mathcal{M}(\mathrm{F}(v))$ whenever $\mathcal{M}(u)=\mathcal{M}(v)$. Therefore, the function $f_{S}: S_{N}^{\oplus} \rightarrow S^{\oplus}$ which 
sends $v$ to $\mathcal{M}\left(\mathrm{F}\left(u_{v}\right)\right)$, for $u_{v}$ any linearization of $v$, is a well defined monoid homomorphism. Moreover, $f_{S}$ does not depend on the chosen representative of $\mathrm{K}$, for if $\mathrm{F} R \mathrm{G}$ then, for all $u \in S^{\otimes}$, there is a symmetry $\sigma_{u}: \mathrm{F}(u) \rightarrow \mathrm{G}(u)$, whence $\mathcal{M}(\mathrm{F}(u))=\mathcal{M}(\mathrm{G}(u))$. Concerning the transitions, consider $f_{T}: T_{N} \rightarrow T$ defined as $f_{T}(t)=\tau$, where $\tau$ is the transition of $C$ corresponding via $\mathrm{F}$ to the transition $\left\{t_{u, v}\right\}$ of $Q(N)$. By Lemma 3.14, $f_{T}$ is well defined, and by Lemma 3.15, it does not depend on the representative of K. Moreover, since $f_{T}\left(t: \mathrm{v} \rightarrow \mathrm{v}^{\prime}\right)=\tau$ implies that $\tau: \pi_{\mathrm{C}, f_{S}(v), f_{S}\left(v^{\prime}\right)}^{0} \dot{\rightarrow} \pi_{\mathrm{C}, f_{S}(v), f_{S}\left(v^{\prime}\right)}^{1}$, we have that $f=\left\langle f_{T}, f_{S}\right\rangle: N \rightarrow \mathcal{N}(\mathrm{C})$ is a morphism in Petri. We have to prove that $\varepsilon_{\mathrm{C}} \circ Q(f)=\mathrm{K}$ in SPetriCat. Without loss of generality, exploiting the fact that $\mathcal{R}$ is a congruence, we prove that $\varepsilon \circ \mathrm{G}=\mathrm{F}$ for chosen representatives $\varepsilon$ of $\varepsilon_{\mathrm{C}}, \mathrm{G}$ of $Q(f)$, and $\mathrm{F}$ of $\mathrm{K}$. In particular, we can assume that $\varepsilon$ is the identity on the objects and that $\mathrm{G}(u)=\mathrm{F}(u)$ for all $u \in S_{N}^{\otimes}$. Then, $\varepsilon \mathrm{G}\left(t_{u, v}\right)=\varepsilon\left([f(t)]_{\mathrm{G}(u), \mathrm{G}(v)}\right)=f(t)_{\mathrm{G}(u), \mathrm{G}(v)}=\tau_{\mathrm{F}(u), \mathrm{F}(v)}=\mathrm{F}\left(t_{u, v}\right)$, the last equality following from $\tau$ being the transition of $C$ corresponding to $\left\{t_{u, v}\right\}$ via $F$. The required equality of functors follows now directly from Definition 3.1. Finally, the uniqueness of $f$ follows immediately, since if the diagram has to commute, then both the definitions of $f_{T}$ and $f_{S}$ are forced.

By general results in category theory, the component $\eta_{N}: N \rightarrow \mathcal{N} Q(N)$ of the unit of the adjunction $Q(-) \dashv \mathcal{N}(-)$ is the unique arrow which makes the diagram commute when $C$ is $Q(N)$ and $\mathrm{K}$ is the (equivalence class of the) identity of $Q(-)$. Applying the previous part of the proof, we have that $\eta_{N}=f$, where $f_{S}$ is the identity of $S_{N}^{\oplus}$ and $f_{T}$ sends $t \in T_{N}$ to $\left\{t_{u, v}\right\} \in T_{\mathcal{X}(\mathcal{Q}(N)}$. Since by the definitions of $\mathcal{N}(-)$ and of transition of $\mathcal{Q}(N)$ we know that $f_{T}$ is an isomorphism, we conclude that $\eta_{N}$ is such.

Finally, we can identify the replete image of $Q_{(-)}$in SPetriCat, i.e., we can identify those symmetric Petri categories which are isomorphic to $Q(N)$, for some net $N$.

Proposition 3.19. (Petri $\cong$ FSPetriCat) Let FSPetriCat be the full subcategory of SPetriCat consisting of those symmetric Petri categories $C$ whose arrows can be generated by tensor and composition from symmetries, and components of transitions of $\mathrm{C}$, uniquely up to the axioms of symmetric strict monoidal categories, i.e., axioms (1) and (2), and the naturality of transitions, i.e., axiom $(\Phi)$.

Then, FSPetriCat and Petri are equivalent via $\mathcal{N}\left({ }_{-}\right)$and $Q\left({ }_{-}\right)$.

Proof. By general results in category theory, it is enough to show that $\mathrm{C}$ belongs to FSPetriCat if and only if the component $\varepsilon_{C}: Q \mathcal{N}(C) \rightarrow C$ of the counit of $Q(-) \dashv \mathcal{N}(-)$ is an isomorphism. Let $\varepsilon$ be a representative of $\varepsilon_{C}$. Clearly, $\varepsilon_{C}$ is iso if and only if $\varepsilon$ is such. Moreover, since $\varepsilon$ is an isomorphism on the objects, it is iso if and only if it is an isomorphism on each homset. Then the result follows, since each arrow of $C$ can be written as tensor and composition of symmetries and component of transitions if and only if $\varepsilon$ is surjective on each homset, and this can be done uniquely (up to the equalities that necessarily hold in any symmetric Petri category) if and only if $\varepsilon$ is injective on each homset.

\section{Strongly Concatenable Processes}

In this section we introduce a slight refinement of concatenable processes and we show that they are abstractly represented by the arrows of the category $Q(N)$. In other words, we find a process-like representation for the arrows of $Q(N)$. This yields a functorial construction for the 

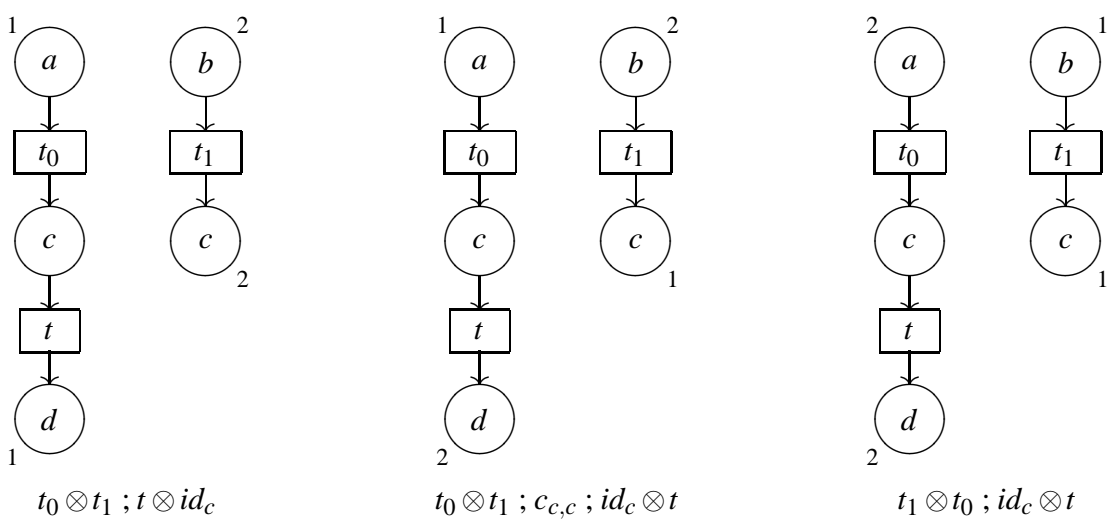

Figure 6. Three strongly concatenable processes corresponding to $\pi$ of Figure 2

category of the processes of a net $N$. Our task is simplified by the work already done in the proof of Proposition 3.6.

Definition 4.1. (Strongly Concatenable Processes) A strongly concatenable process of a net $N$ is a triple $(\pi, \ell, L)$ where $\pi: \Theta \rightarrow N$ is a process of $N$, and $\ell$ and $L$ are total orderings of, respectively, the minimal and the maximal places of $\Theta$, i.e., bijections $\ell: \min (\Theta) \rightarrow\{1, \ldots,|\min (\Theta)|\}$ and $L: \max (\Theta) \rightarrow\{1, \ldots,|\max (\Theta)|\}$.

An isomorphism of strongly concatenable processes is an isomorphism of the underlying processes which, in addition, preserves the orderings $\ell$ and $L$. As usual, we identify isomorphic strongly concatenable processes.

Example 4.2. Thus, as already remarked, a strongly concatenable process is simply a GoltzReisig process whose minimal and maximal places are linearly ordered. As in the case of concatenable processes, we shall represent strongly concatenable processes by decorating places when necessary - with the values of $\ell$ and $L$. As an example, Figure 6 shows three (of the four) different strongly concatenable processes obtained by linearizing source and target of the concatenable process $\pi$ of Figure 2. The corresponding arrows of $Q(N)$ are also given; the reader can safely ignore them for the moment: the correspondence will become clear after the proof of our representation result in Proposition 4.5.

As in the case of concatenable processes, strongly concatenable processes of $N$ can be endowed with a symmetric monoidal algebraic structure, actually rather similar to the one of $\mathcal{C P}(N)$. First, we associate a source and a target in $S_{N}^{\otimes}$ to each strongly concatenable process by taking the string corresponding to the linear ordering of, respectively, $\min (\Theta)$ and $\max (\Theta)$. Then, the concatenation of $\left(\pi_{0}: \Theta_{0} \rightarrow N, \ell_{0}, L_{0}\right): u \rightarrow v$ and $\left(\pi_{1}: \Theta_{1} \rightarrow N, \ell_{1}, L_{1}\right): v \rightarrow w$ is the concatenable process $(\pi: \Theta \rightarrow N, \ell, L): u \rightarrow w$ defined as follows (see also Figure 7), where + denotes the disjoint union of sets and functions.

- Let $m$ be the cardinality of $\max \left(\Theta_{0}\right)$ and $\min \left(\Theta_{1}\right)$, coinciding by definition of source and target, and consider the functions $\bar{L}:\{1, \ldots, m\} \rightarrow S_{\Theta_{0}}$ and $\bar{\ell}:\{1, \ldots, m\} \rightarrow S_{\Theta_{1}}$ defined by

$$
\bar{L}=\{1, \ldots, m\} \stackrel{L_{0}^{-1}}{\longrightarrow} \max \left(\Theta_{0}\right) \hookrightarrow S_{\Theta_{0}} \quad \text { and } \quad \bar{\ell}=\{1, \ldots, m\} \stackrel{\ell_{1}^{-1}}{\longrightarrow} \min \left(\Theta_{1}\right) \hookrightarrow S_{\Theta_{1}} .
$$




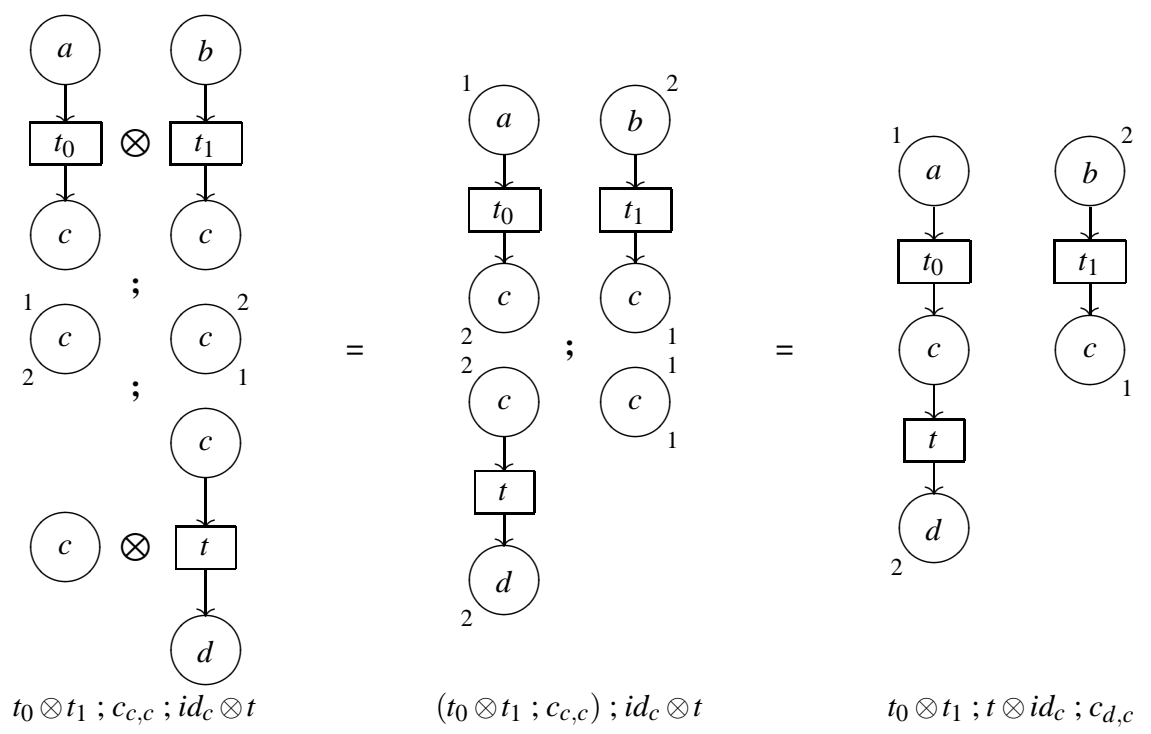

Figure 7. An example of the algebra of strongly concatenable processes

Let $S$ be a pushout of $\bar{L}$ and $\bar{\ell}$, e.g., $S_{\Theta_{0}} \cup S_{\Theta_{1}}$ quotiented by the equations $\bar{L}(i)=\bar{\ell}(i)$, for $i=1, \ldots, m$, when $S_{\Theta_{0}}$ and $S_{\Theta_{1}}$ are disjoint, and let $i n_{0}: S_{\Theta_{0}} \hookrightarrow S$ and $i n_{1}: S_{\Theta_{1}} \hookrightarrow S$ be the corresponding injections. Then,

$$
\Theta=\left(\partial_{\Theta_{0}}^{0}+\partial_{\Theta_{1}}^{0}, \partial_{\Theta_{0}}^{1}+\partial_{\Theta_{1}}^{1}: T_{\Theta_{0}}+T_{\Theta_{1}} \rightarrow S^{\oplus}\right),
$$

where $\partial_{\Theta_{i}}^{j}$ is $T_{\Theta} \stackrel{\partial_{\Theta_{i}}^{j}}{\longrightarrow} S_{\Theta_{i}}^{\oplus} \stackrel{i n_{i}^{\oplus}}{\longrightarrow} S^{\oplus}$ and $i n_{i}^{\oplus}$ is the free monoid homomorphism induced by $i n_{i}$.

- Let $g_{0}: S_{\Theta_{0}} \rightarrow S_{N}$ and $g_{1}: S_{\Theta_{1}} \rightarrow S_{N}$ be the functions underlying the place components of, respectively, $\pi_{0}$ and $\pi_{1}$. Since $g_{0} \circ \bar{L}=g_{1} \circ \bar{\ell}$, there exists a (unique) function $g: S \rightarrow S_{N}$ (induced by the pushout) such that $g \circ i n_{0}=g_{0}$ and $g \circ i n_{1}=g_{1}$, i.e., that agrees with $\pi_{0}$ on $S_{\Theta_{0}}$ and with $\pi_{1}$ on $S_{\Theta_{1}}$. Then $\pi=\left\langle\pi_{0 T}+\pi_{1 T}, g^{\oplus}\right\rangle$.

$-\ell\left(\operatorname{in}_{0}(a)\right)=\ell_{0}(a)$.

- $L\left(\operatorname{in}_{1}(a)\right)=L_{1}(a)$.

Proposition 4.3. (The Category $C Q(N)$ ) Under the above defined operation of sequential composition, the strongly concatenable processes of $N$ form a category $C Q(N)$ with identities those processes consisting only of places, which therefore are both minimal and maximal, and such that $\ell=L$.

Concerning the operation of parallel composition, for $S C P_{0}=\left(\pi_{0}: \Theta_{0} \rightarrow N, \ell_{0}, L_{0}\right): u \rightarrow v$ and $S C P_{1}=\left(\pi_{1}: \Theta_{1} \rightarrow N, \ell_{1}, L_{1}\right): u^{\prime} \rightarrow v^{\prime}$ strongly concatenable processes, $S C P_{0} \otimes S C P_{1}$ is the process $(\pi: \Theta \rightarrow N, \ell, L): u \otimes u^{\prime} \rightarrow v \otimes v^{\prime}$ defined below (see also Figure 7), where $i n_{i}$ denotes the inclusion of $S_{\Theta_{i}}$ in the disjoint union $S_{\Theta_{0}}+S_{\Theta_{1}}$.

$-\Theta=\left(\partial_{\Theta_{0}}^{0}+\partial_{\Theta_{1}}^{0}, \partial_{\Theta_{0}}^{1}+\partial_{\Theta_{1}}^{1}: T_{\Theta_{0}}+T_{\Theta_{1}} \rightarrow\left(S_{\Theta_{0}}+S_{\Theta_{1}}\right)^{\oplus}\right) ;$

$-\pi=\left\langle\pi_{0 T}+\pi_{1 T}, \pi_{0 S}+\pi_{1 S}\right\rangle$;

- $\ell\left(\operatorname{in}_{0}(a)\right)=\ell_{0}(a) \quad$ and $\quad \ell\left(\operatorname{in}_{1}(a)\right)=\left|\min \left(\Theta_{0}\right)\right|+\ell_{1}(a)$;

$-L\left(\operatorname{in}_{0}(a)\right)=L_{0}(a)$ and $L\left(i_{1}(a)\right)=\left|\max \left(\Theta_{0}\right)\right|+L_{1}(a)$. 

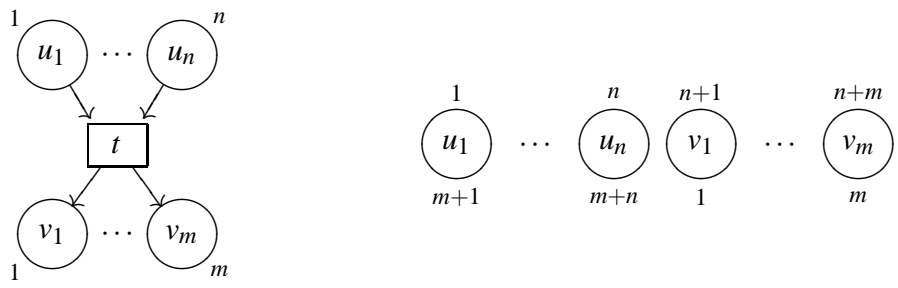

Figure 8. A transitions $t_{u, v}: u \rightarrow v$ and the symmetry $\bar{\gamma}(u, v)$ in $\mathcal{C} Q(N)$

It is easy to verify that $\otimes$ is a functor $\otimes: C Q(N) \times C Q(N) \rightarrow C Q(N)$. The strongly concatenable processes consisting only of places are analogous in $C Q(N)$ to the symmetries of $Q(N)$. In particular, for any $u, v \in S^{\otimes}$, the strongly concatenable process $\bar{\gamma}(u, v)$ consisting of places are in one-to-one correspondence with the elements of the string $u \otimes v$ mapped by $\pi$ to the corresponding places of $N$, and such that $\ell\left(u_{i}\right)=i, \ell\left(v_{i}\right)=|u|+i, L\left(u_{i}\right)=|v|+i$, and $L\left(v_{i}\right)=i$, plays in $C Q(N)$ the role played by the symmetry $c_{u, v}$, i.e., the permutation $\gamma(u, v)$, in $Q(N)$ (see also Figure 8).

Proposition 4.4. (The Symmetric Monoidal Category $C Q(N)$ ) Under the above defined tensor product, $C Q(N)$ is a symmetric strict monoidal category whose symmetry isomorphism is the

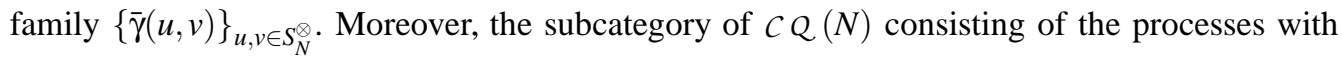
only places is the category of symmetries of $C Q(N)$ and is isomorphic to $S_{y} m_{N}^{*}$.

Proof. Concerning the first claim, it is enough to verify that $\mathcal{C} Q(N)$ satisfies the axioms (6) with respect to $\otimes$ and the symmetries $\bar{\gamma}(u, v)$ defined above. The task is really immediate and thus omitted.

Let $S y m$ be the subcategory of the processes consisting only of places of $\mathcal{C}(N)$. Since $\otimes$ restricts to a functor $S y m \times S y m \rightarrow S y m$, we have that $S y m$ is a symmetric strict monoidal category

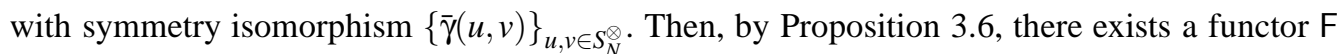
from $S y m_{N}^{*}$ to $S y m$, corresponding to the identity function on $S_{N}^{\otimes}$, which is the identity on the objects and such that $\mathrm{F}(\gamma(u, v))=\bar{\gamma}(u, v)$. Moreover, since for any $u, v \in S_{N}^{\otimes}$ the strongly concatenable processes from $u$ to $v$ in Sym are clearly isomorphic to the permutations $p: u \rightarrow v$ in $\operatorname{Sym}_{N}^{*}$, it follows easily that $\mathrm{F}$ is full and faithful. Therefore, $\mathrm{F}$ is an isomorphism. This means that Sym is generated via composition and tensor product from the symmetries $\bar{\gamma}(u, v)$ and from the identities, i.e., that $S y m$ is the category of symmetries of $C Q(N)$.

The transitions $t$ of $N$ are faithfully represented in the obvious way by processes with a unique transition which is in the post-set of any minimal place and in the pre-set of any maximal place, minimal and maximal places being in one-to-one correspondence, respectively, with $\partial_{N}^{0}(t)$ and $\partial_{N}^{1}(t)$. Thus, varying $\ell$ and $L$ on the process corresponding to a transition we obtain a representative in $C Q(N)$ of each instance $t_{u, v}$ of $t$ in $Q(N)$ (see also Figure 8).

We can show the announced correspondence between $C Q(N)$ and $Q(N)$.

Proposition 4.5. (Strongly Concatenable Processes vs. $Q(-)) \mathcal{Q}(N)$ and $Q(N)$ are isomorphic in SSMC.

Proof. First observe that $\mathcal{C} Q(N)$ satisfies axiom $(\Phi)$ of Definition 3.1, the symmetries and the (instances of) transitions being as explained above. In order to prove this statement, let 
$T_{u, v}=\left(\pi_{0}: \Theta_{0} \rightarrow N, \ell_{0}, L_{0}\right)$ and $T_{u^{\prime}, v^{\prime}}=\left(\pi_{1}: \Theta_{1} \rightarrow N, \ell_{1}, L_{1}\right)$ be different instances of some transition $t$, and let $S: u \rightarrow u^{\prime}$ and $S^{\prime}: v \rightarrow v^{\prime}$ be symmetries of $C Q(N)$. Assume also that $S^{-1}$ and $S^{\prime}$ correspond, respectively, to the permutations $p: u^{\prime} \rightarrow u$ and $q: v \rightarrow v^{\prime}$ in $Q(N)$. Then, $S^{-1} ; T_{u, v} ; S$ is (isomorphic to) $\left(\pi_{0}: \Theta_{0} \rightarrow N, p \circ \ell_{0}, q \circ L_{0}\right)$. Consider now the function $g: S_{\Theta_{0}} \rightarrow S_{\Theta_{1}}$ such that $g(x)=\ell_{1}^{-1}\left(p\left(\ell_{0}(x)\right)\right)$ if $x \in \min \left(\Theta_{0}\right)$ and $g(x)=L_{1}^{-1}\left(q\left(L_{0}(x)\right)\right)$ if $x \in \max \left(\Theta_{0}\right)$. Clearly, by definition of $\Theta_{0}$ and $\Theta_{1}, g$ is an isomorphism. Moreover, since for each $x \in \min \left(\Theta_{0}\right)$ and $y \in \max \left(\Theta_{0}\right)$ we have that $u_{\ell_{0}(x)}=u_{p\left(\ell_{0}(x)\right)}^{\prime}$ and $v_{L_{0}(y)}=u_{q\left(L_{1}(y)\right)}^{\prime}$, it follows that $\pi_{1}(g(x))=$ $u_{\ell_{1}(g(x))}^{\prime}=u_{p\left(\ell_{0}(x)\right)}^{\prime}=u_{\ell_{0}(x)}=\pi_{0}(x)$ and that $\pi_{1}(g(y))=u_{L_{1}(g(y))}^{\prime}=u_{q\left(L_{0}(y)\right)}^{\prime}=u_{L_{0}(y)}=\pi_{0}(y)$. Therefore, we have an isomorphism $\left\langle f, g^{\oplus}\right\rangle: \Theta_{0} \rightarrow \Theta_{1}$, where $f$ is the function which maps the unique transition in $\Theta_{0}$ to the unique transition in $\Theta_{1}$. Then, $S^{-1} ; T_{u, v} ; S^{\prime}=T_{u^{\prime}, v^{\prime}}$, i.e., ( $\left.\Phi\right)$ holds.

Thus, since $Q(N)$ is the free symmetric strict monoidal category built on $S y m_{N}^{*}$ plus the additional arrows in $T_{N}$ and which satisfies axiom $(\Phi)$, there is a strict symmetric monoidal functor $\mathcal{H}: Q(N) \rightarrow C Q(N)$ which is the identity on the objects and sends the generators, i.e., symmetries and transitions, to the corresponding strongly concatenable processes. We want to show that $\mathcal{H}$ is an isomorphism. Observe that, by Proposition 4.4, we already know that $\mathcal{H}$ is an isomorphism between the corresponding categories of symmetries.

fullness. It is completely trivial to see that any strongly concatenable process $S C P$ may be obtained as a concatenation $S C P_{0} ; \ldots ; S C P_{n}$ of strongly concatenable processes $S C P_{i}$ of depth one. Now, each of these $S C P_{i}$ may be split into the concatenation of a symmetry $S_{0}^{i}$, the tensor of the (processes representing the) transitions which appear in it plus some identities, say $u_{i} \otimes \bigotimes_{j} T_{j}^{i}$ and finally another symmetry $S_{1}^{i}$. In fact, we can take the tensor of the transitions which appear in $S C P_{i}$ in any order, and multiply the result by an identity concatenable process in order to get the correct source and target; we then need a pre-concatenation and a post-concatenation with symmetries in order to get the right indexing of minimal and maximal places. Then, we obtain

$$
S C P=S_{0}^{0} ;\left(u_{1} \otimes \bigotimes_{j} T_{j}^{1}\right) ;\left(S_{1}^{0} ; S_{0}^{1}\right) ; \ldots ;\left(S_{1}^{n-1} ; S_{0}^{n}\right) ;\left(u_{n} \otimes \bigotimes_{j} T_{j}^{n}\right) ; S_{1}^{n},
$$

which shows that every strongly concatenable process is in the image of $\mathcal{H}$.

faithfulness. The arrows of $Q(N)$ are equivalence classes, modulo the axioms stated in Definition 3.1, of terms built by applying tensor and sequential composition to the identities $i d_{u}$, the symmetries $c_{u, v}$, and the transitions $t_{u, v}$. We have to show that, given two such terms $\alpha$ and $\beta$, whenever $\mathcal{H}(\alpha)=\mathcal{H}(\beta)$ we have $\alpha={ }_{\mathcal{E}} \beta$, where $=_{\mathcal{E}}$ is the equivalence induced by the axioms (1), (2) and $(\Phi)$.

First of all, observe that if $\mathcal{H}(\alpha)$ is a strongly concatenable process $S C P$ of depth $n$, then $\alpha$ can be proved equal to a term

$$
\alpha^{\prime}=s_{0} ;\left(i d_{u_{1}} \otimes \bigotimes_{j} \tau_{j}^{1}\right) ; s_{1} ; \ldots ; s_{n-1} ;\left(i d_{u_{n}} \otimes \bigotimes_{j} \tau_{j}^{n}\right) ; s_{n}
$$

where, for $1 \leq i \leq n, \tau_{j}^{i}=\left(t_{j}^{i}\right)_{u_{j}^{i}, v_{j}^{i}}$ and the transitions $t_{j}^{i}$, for $1 \leq j \leq n_{i}$, are exactly the transitions of $S C P$ at depth $i$ and where $s_{0}, \ldots, s_{n}$ are symmetries. Moreover, we can assume that in the $i$ th tensor product $\bigotimes_{j} \tau_{j}^{i}$ the transitions are indexed according to a global ordering $\leq$ of $T_{N}$ assumed for the purpose of this proof, i.e., $t_{1}^{i} \leq \cdots \leq t_{n_{i}}^{i}$, for $1 \leq i \leq n$. Our claim can easily be shown by induction on the structure of terms. In fact, using axioms (1) $\alpha$ can be rewritten as $\alpha_{1} ; \ldots ; \alpha_{h}$, where $\alpha_{i}=\bigotimes_{k} \xi_{k}^{i}$ and $\xi_{k}^{i}$ is either a transition or a symmetry. Now, observe that, by functoriality of $\otimes$, for any $\alpha^{\prime}: u^{\prime} \rightarrow v^{\prime}, \alpha^{\prime \prime}: u^{\prime \prime} \rightarrow v^{\prime \prime}$ and $s: u \rightarrow u$, we have 
$\alpha^{\prime} \otimes s \otimes \alpha^{\prime \prime}=\left(i d_{u^{\prime}} \otimes s \otimes i d_{u^{\prime \prime}}\right) ;\left(\alpha^{\prime} \otimes i d_{u} \otimes \alpha^{\prime \prime}\right)$, and thus, by repeated applications of (1), we can prove that $\alpha$ is equivalent to $\bar{s}_{0} ; \bar{\alpha}_{1} ; \bar{s}_{1} \ldots ; \bar{s}_{h-1} ; \bar{\alpha}_{h}$, where $\bar{s}_{0}, \ldots, \bar{s}_{h-1}$ are symmetries and each $\bar{\alpha}_{i}$ is a tensor $\bigotimes_{k} \bar{\xi}_{k}^{i}$ of transitions and identities. The fact that the transitions at depth $i$ can be brought to the $i$ th tensor product, follows intuitively from the facts that they are 'disjointly enabled', i.e., concurrent to each other, and that they depend causally on some transition at depth $i-1$. In particular, the sources of the transitions of depth 1 can be target only of symmetries. Therefore, reasoning formally as above, they can be pushed up to $\bar{\alpha}_{1}$ exploiting axioms (1). Then, the same happens for the transitions of depth 2, which can be brought to $\bar{\alpha}_{2}$. Proceeding in this way, eventually we show that $\alpha$ is equivalent to the composition $\overline{\bar{s}}_{0} ; \overline{\bar{\alpha}}_{1} ; \overline{\bar{s}}_{1} \ldots ; \overline{\bar{s}}_{n-1} ; \overline{\bar{\alpha}}_{n} ; \overline{\bar{s}}_{n}$ of the symmetries $\overline{\bar{s}}_{0}, \ldots, \overline{\bar{s}}_{n}$ and the products $\overline{\bar{\alpha}}_{i}=\bigotimes_{k} \overline{\bar{\xi}}_{k}^{i}$ of transitions at depth $i$ and identities. Finally, the order of the $\overline{\bar{\xi}}_{k}^{i}$ can be permuted in the way required by $\leq$. This is achieved by preand post-composing each product by appropriate interchange symmetries. More precisely, let $\sigma$ be a permutation such that $\bigotimes_{k} \overline{\bar{\xi}}_{i_{\sigma(k)}}$ coincides with $i d_{u_{i}} \otimes \bigotimes_{j} \tau_{j}^{i}$, suppose that $\overline{\bar{\xi}} i_{k}: u_{k}^{i} \rightarrow v_{k}^{i}$, for $1 \leq k \leq k_{i}$. Then, by definition of interchange permutation in $S y m_{N}^{*}$, we have that

$$
\sigma\left(u_{1}^{i}, \ldots, u_{k_{i}}^{i}\right) ;\left(\bigotimes_{k} \overline{\bar{\xi}}_{\sigma(k)}^{i}\right)=\left(\bigotimes_{k} \overline{\bar{\xi}}_{k}^{i}\right) ; \sigma\left(v_{1}^{i}, \ldots, v_{k_{i}}^{i}\right)
$$

and then, since $\sigma\left(u_{1}^{i}, \ldots, u_{k_{i}}^{i}\right)$ is an isomorphism, we have that

$$
\left(i d_{u_{i}} \otimes \bigotimes_{j} \tau_{j}^{i}\right)=\sigma\left(u_{1}^{i}, \ldots, u_{k_{i}}^{i}\right)^{-1} ;\left(\bigotimes_{k} \overline{\bar{\xi}}_{k}^{i}\right) ; \sigma\left(v_{1}^{i}, \ldots, v_{k_{i}}^{i}\right) .
$$

Now, applying the same argument to $\beta$, one can prove that it is equivalent to a term $\beta^{\prime}=$ $p_{0} ; \beta_{0} ; p_{1} ; \ldots p_{n-1} ; \beta_{n} ; p_{n}$, where $p_{0}, \ldots, p_{n}$ are symmetries and $\beta_{i}$ is the product of (instances of) the transitions at depth $i$ in $\mathcal{H}(\beta)$ and of identities. Then, since $\mathcal{H}(\alpha)=\mathcal{H}(\beta)$, and since the transitions occurring in $\beta_{i}$ are indexed in a predetermined way, we conclude that $\beta_{i}=\left(i d_{u_{i}} \otimes \otimes_{j} \bar{\tau}_{j}^{i}\right)$, where $\bar{\tau}_{j}^{i}=\left(t_{j}^{i}\right)_{\bar{u}_{j}^{i}, \bar{v}_{j}^{i}}$ i.e.,

$$
\begin{aligned}
& \alpha^{\prime}=s_{0} ;\left(i d_{u_{1}} \otimes \bigotimes_{j}\left(t_{j}^{1}\right)_{u_{j}^{1}, v_{j}^{1}}\right) ; s_{1} ; \ldots ; s_{n-1} ;\left(i d_{u_{n}} \otimes \bigotimes_{j}\left(t_{j}^{n}\right)_{u_{j}^{n}, v_{j}^{n}}\right) ; s_{n} \\
& \beta^{\prime}=p_{0} ;\left(i d_{u_{1}} \otimes \bigotimes_{j}\left(t_{j}^{1}\right)_{\bar{u}_{j}^{1}, \bar{v}_{j}^{1}}\right) ; p_{1} ; \ldots ; p_{n-1} ;\left(i d_{u_{n}} \otimes \bigotimes_{j}\left(t_{j}^{n}\right)_{\bar{u}_{j}^{n}, \bar{v}_{j}^{n}}\right) ; p_{n} .
\end{aligned}
$$

In other words, the only possible differences between $\alpha^{\prime}$ and $\beta^{\prime}$ are the symmetries and the sources and targets of the corresponding instances of transitions. Observe now that the steps which led from $\alpha$ to $\alpha^{\prime}$ and from $\beta$ to $\beta^{\prime}$ have been performed by using the axioms which define $Q(N)$ and since such axioms hold in $\mathcal{C} Q(N)$ as well and $\mathcal{H}$ preserves them, we have that $\mathcal{H}\left(\alpha^{\prime}\right)=\mathcal{H}(\alpha)=\mathcal{H}(\beta)=\mathcal{H}\left(\beta^{\prime}\right)$. Thus, we conclude the proof by showing that, if $\alpha$ and $\beta$ are terms of the form given in (5) which differ only by the intermediate symmetries and if $\mathcal{H}(\alpha)=\mathcal{H}(\beta)$, then $\alpha$ and $\beta$ are equal in $Q(N)$.

We proceed by induction on $n$. Observe that if $n$ is zero then there is nothing to show: since we know that $\mathcal{H}$ is an isomorphism on the symmetries, $s_{0}$ and $p_{0}$, and thus $\alpha$ and $\beta$, must coincide. To provide a correct base for the induction, we need to prove the thesis also for $n=1$.

depth 1. In this case, we have

$$
\begin{aligned}
& \alpha=s_{0} ;\left(i d_{u} \otimes \bigotimes_{j}\left(t_{j}\right)_{u_{j}, v_{j}}\right) ; s_{1} \\
& \beta=p_{0} ;\left(i d_{u} \otimes \otimes_{j}\left(t_{j}\right)_{\bar{u}_{j}, \bar{v}_{j}}\right) ; p_{1} .
\end{aligned}
$$


Without loss of generality we may assume that $p_{0}$ and $p_{1}$ are identities. In fact, we can multiply both terms by $p_{0}^{-1}$ on the left and by $p_{1}^{-1}$ on the right and obtain a pair of terms whose images through $\mathcal{H}$ still coincide and whose equality implies the equality in $Q(N)$ of the original $\alpha$ and $\beta$. Let $(\pi: \Theta \rightarrow N, \ell, L)$ and $(\bar{\pi}: \bar{\Theta} \rightarrow N, \bar{\ell}, \bar{L})$ be, respectively, the strongly concatenable processes $\mathcal{H}\left(i d_{u} \otimes \bigotimes_{j}\left(t_{j}\right)_{u_{j}, v_{j}}\right)$ and $\mathcal{H}\left(i d_{u} \otimes \bigotimes_{j}\left(t_{j}\right)_{\bar{u}_{j}, \bar{v}_{j}}\right)$. Clearly, we can assume that $\mathcal{H}\left(s_{0}\right)$ and $\mathcal{H}\left(s_{1}\right)$ are respectively $\left(\pi_{0}: \Theta_{0} \rightarrow N, \ell^{\prime}, \ell\right)$ and $\left(\pi_{1}: \Theta_{1} \rightarrow N, L, L^{\prime}\right)$, where $\Theta_{0}$ is $\min (\Theta), \Theta_{1}$ is $\max (\Theta)$, $\pi_{0}$ and $\pi_{1}$ are the corresponding restrictions of $\pi$, and $\ell^{\prime}$ and $L^{\prime}$ are the orderings respectively of the minimal and the maximal places of $\Theta$.

Then, we have that $\mathcal{H}\left(s_{0} ;\left(i d_{u} \otimes \bigotimes_{j}\left(t_{j}\right)_{u_{j}, v_{j}}\right) ; s_{1}\right)$ is $\left(\pi: \Theta \rightarrow N, \ell^{\prime}, L^{\prime}\right)$, and by hypothesis there is an isomorphism $\varphi: \Theta \rightarrow \bar{\Theta}$ such that $\bar{\pi} \circ \varphi=\pi$ and which respects all the orderings, i.e., $\bar{\ell}(\varphi(a))=\ell^{\prime}(a)$ and $\bar{L}(\varphi(b))=L^{\prime}(b)$, for all $a \in \Theta_{0}$ and $b \in \Theta_{1}$. Let us write $i d_{u} \otimes \bigotimes_{j}\left(t_{j}\right)_{u_{j}, v_{j}}$ as $\bigotimes_{k} \xi_{k}$ and $i d_{u} \otimes \bigotimes_{j}\left(t_{j}\right)_{\bar{u}_{j}, \bar{v}_{j}}$ as $\bigotimes_{k} \bar{\xi}_{k}$, where $\xi_{k}$, respectively $\bar{\xi}_{k}$, is either a transition $\left(t_{j}\right)_{u_{j}, v_{j}}$, respectively $\left(t_{j}\right)_{\bar{u}_{j}, \bar{v}_{j}}$, or the identity of a place in $u$. Clearly, $\varphi$ induces a permutation, namely the permutation $\sigma$ such that $\bar{\xi}_{\sigma(k)}=\varphi\left(\xi_{k}\right)$. In order for $\varphi$ to be a morphism of nets, it must map the (places corresponding to the) pre-set, respectively post-set, of $\left(t_{j}\right)_{u_{j}, v_{j}}$ to (the places correspond-

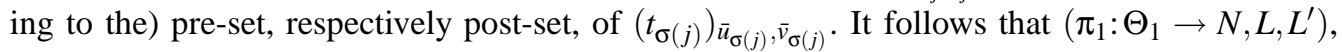
which is $\mathcal{H}\left(s_{1}\right)$, must be a symmetry obtained by post-concatenating the image via $\mathcal{H}$ of the interchange symmetry $\sigma\left(\bar{v}_{1}, \ldots, \bar{v}_{k_{i}}\right)$ in $C Q(N)$ with a tensor product $\otimes_{j} S_{j}^{1}$ of symmetries, one for each $t$ occurring in $\alpha$, where $S_{j}^{1}: v_{j} \rightarrow \bar{v}_{j}$, whose role is to reorganize the tokens in the postsets of each transitions. Reasoning along the same lines, we can conclude that $\left(\pi_{0}: \Theta_{0} \rightarrow N, \ell, \ell^{\prime}\right)$, which is $\mathcal{H}\left(s_{0}\right)^{-1}$, must be a symmetry obtained by concatenating a tensor product $\bigotimes_{j} S_{j}^{0}$, where $S_{j}^{0}: u_{j} \rightarrow \bar{u}_{j}$ is a symmetry, with the image via $\mathcal{H}$ of the interchange symmetry $\sigma\left(\bar{u}_{1}, \ldots, \bar{u}_{k_{i}}\right)$. Then, since $\mathcal{H}$ is an isomorphism between $\operatorname{Sym}_{Q(N)}$ and $\operatorname{Sym}_{\mathcal{L Q}(N)}, s_{0}$ and $s_{1}$ must necessarily be, respectively, $\sigma\left(\bar{u}_{1}, \ldots, \bar{u}_{k_{i}}\right)^{-1} ;\left(i d_{u} \otimes \bigotimes_{j} s_{j}^{0}\right)$, and $\left(i d_{u} \otimes \bigotimes_{j} s_{j}^{1}\right) ; \sigma\left(\bar{v}_{1}, \ldots, \bar{v}_{k_{i}}\right)$, where $s_{j}^{0}: \bar{u}_{j} \rightarrow u_{j}$ and $s_{j}^{1}: v_{j} \rightarrow \bar{v}_{j}$ are symmetries.

Then, by distributing the tensor of symmetries on the transitions and using $(\Phi)$, we show that

$$
\begin{aligned}
\alpha & =\sigma\left(\bar{u}_{1}, \ldots, \bar{u}_{k_{i}}\right)^{-1} ;\left(i d_{u} \otimes \bigotimes_{j} s_{j}^{0} ;\left(t_{j}\right)_{u_{j}, v_{j}} ; s_{j}^{1}\right) ; \sigma\left(\bar{v}_{1}, \ldots, \bar{v}_{k_{i}}\right) \\
& =\sigma\left(\bar{u}_{1}, \ldots, \bar{u}_{k_{i}}\right)^{-1} ;\left(i d_{u} \otimes \bigotimes_{j}\left(t_{j}\right)_{\bar{u}_{j}, \bar{v}_{j}}\right) ; \sigma\left(\bar{v}_{1}, \ldots, \bar{v}_{k_{i}}\right),
\end{aligned}
$$

which, by definition of interchange symmetry, is $\left(i d_{u} \otimes \otimes_{j}\left(t_{j}\right)_{\bar{u}_{j}, \bar{v}_{j}}\right)$. Thus, we have $\alpha=_{\mathcal{E}} \beta$ as required.

Inductive step. Suppose that $n>1$ and let $\alpha=\alpha^{\prime} ; \alpha^{\prime \prime}$ and $\beta=\beta^{\prime} ; \beta^{\prime \prime}$, where

$$
\begin{aligned}
& \alpha^{\prime}=s_{0} ;\left(i d_{u_{1}} \otimes \bigotimes_{j} \tau_{j}^{1}\right) ; s_{1} ; \ldots ; s_{n-1} \quad \text { and } \quad \alpha^{\prime \prime}=\left(i d_{u_{n}} \otimes \bigotimes_{j} \tau_{j}^{n}\right) ; s_{n} \\
& \beta^{\prime}=p_{0} ;\left(i d_{u_{1}} \otimes \bigotimes_{j} \bar{\tau}_{j}^{1}\right) ; p_{1} ; \ldots ; p_{n-1} \quad \text { and } \quad \beta^{\prime \prime}=\left(i d_{u_{n}} \otimes \bigotimes_{j} \bar{\tau}_{j}^{n}\right) ; p_{n}
\end{aligned}
$$

We show that there exists a symmetry $s$ in $Q(N)$ such that $\mathcal{H}\left(\alpha^{\prime} ; s\right)=\mathcal{H}\left(\beta^{\prime}\right)$ and $\mathcal{H}\left(s^{-1} ; \alpha^{\prime \prime}\right)=$ $\mathcal{H}\left(\beta^{\prime \prime}\right)$. Then, by the induction hypothesis, we have $\left(\alpha^{\prime} ; s\right)={ }_{\mathcal{E}} \beta^{\prime}$ and $\left(s^{-1} ; \alpha^{\prime \prime}\right)={ }_{\mathcal{E}} \beta^{\prime \prime}$. Therefore, we conclude that $\left(\alpha^{\prime} ; s ; s^{-1} ; \alpha^{\prime \prime}\right)=_{\mathcal{E}}\left(\beta^{\prime} ; \beta^{\prime \prime}\right)$, i.e., that $\alpha=\beta$ in $Q(N)$.

Let $(\pi: \Theta \rightarrow N, \ell, L)$ be the strongly concatenable process $\mathcal{H}(\alpha)=\mathcal{H}(\beta)$. Without loss of generality we may assume that the strongly concatenable processes $\mathcal{H}\left(\alpha^{\prime}\right)$ and $\mathcal{H}\left(\beta^{\prime}\right)$ are, respectively, $\left(\pi: \Theta^{\prime} \rightarrow N, \ell^{\prime}, L^{\alpha^{\prime}}\right)$ and $\left(\pi^{\prime}: \Theta^{\prime} \rightarrow N, \ell^{\prime}, L^{\beta^{\prime}}\right)$, where $\Theta^{\prime}$ is the subnet of depth $n-1$ of $\Theta, \ell^{\prime}$ is the appropriate restriction of $\ell$ and finally $L^{\alpha^{\prime}}$ and $L^{\beta^{\prime}}$ are orderings of the places at depth $n-1$ of $\Theta$. Consider the symmetry $S=(\bar{\pi}: \bar{\Theta} \rightarrow N, \bar{\ell}, \bar{L})$ in $C Q(N)$, where 
- $\bar{\Theta}$ is the process net consisting of the maximal places of $\Theta^{\prime}$;

$-\bar{\pi}: \bar{\Theta} \rightarrow N$ is the restriction of $\pi$ to $\bar{\Theta}$;

$-\bar{\ell}=L^{\alpha^{\prime}}$

$-\bar{L}=L^{\beta^{\prime}}$.

Then, by definition, we have $\mathcal{H}\left(\alpha^{\prime}\right) ; S=\mathscr{H}\left(\beta^{\prime}\right)$. Let us consider now $\alpha^{\prime \prime}$ and $\beta^{\prime \prime}$. We can assume that $\mathcal{H}\left(\alpha^{\prime \prime}\right)$ and $\mathcal{H}\left(\beta^{\prime \prime}\right)$ are, respectively, $\left(\pi^{\prime \prime}: \Theta^{\prime \prime} \rightarrow N, \ell^{\alpha^{\prime \prime}}, L^{\prime \prime}\right)$ and $\left(\pi^{\prime \prime}: \Theta^{\prime \prime} \rightarrow N, \ell^{\beta^{\prime \prime}}, L^{\prime \prime}\right)$, where $\Theta^{\prime \prime}$ is the process net obtained by removing from $\Theta$ the subnet $\Theta^{\prime}, L^{\prime \prime}$ is the restriction of $L$ to $\Theta^{\prime \prime}$, and $\ell^{\alpha^{\prime \prime}}$ and $\ell^{\beta^{\prime \prime}}$ are orderings of the places at depth $n-1$ of $\Theta$. Now, in our hypothesis, it must be $L^{\alpha^{\prime}}=\ell^{\alpha^{\prime \prime}}$ and $L^{\beta^{\prime}}=\ell^{\beta^{\prime \prime}}$, which shows directly that $S^{-1} ; \mathcal{H}\left(\alpha^{\prime \prime}\right)=\mathcal{H}\left(\beta^{\prime \prime}\right)$. Then, $s=\mathcal{H}^{-1}(S)$ is the required symmetry of $Q(N)$.

Then, since $\mathcal{H}$ is full and faithful and is an isomorphism on the objects, it is an isomorphism and the proof is concluded.

We conclude this section with a few remarks concerning the relationships between $\mathcal{P}(N)$ and $Q(N)$. As we have already noticed, $Q(N)$ can be quotiented down to $P(N)$ - although not in a functorial way - by imposing the commutativity of the tensor product on the objects and the equations $c_{a, b}=i d$, for $a \neq b \in S_{N}$. On the other hand, there is clearly no reasonable way to map $P(N)$ in $Q(N)$.

At the level of processes, the difference - clearly illustrated by Figures 2, 6 and 7 - is in our opinion not very deep, at least conceptually. Intuitively, while one the one hand $Q(N)$ has many more generators than $P(N)$, on the other hand process composition is a more restricted operation, since source and targets are strings rather than multisets. This is why the difference between concatenable and strongly concatenable processes is completely localized in the orderings of their minimal and maximal places.

Apart from such qualitative considerations, the essential difference between $P(N)$ and $Q(N)$ remains, of course, the symmetry $c_{a, b}$ for $a \neq b \in S_{N}$. To illustrate its role, let us consider again the processes of Figure 6. They all correspond to the concatenable process $\pi$ of Figure 2. In fact, by the second of (2), $\pi_{1}=t_{0} \otimes t_{1} ; t \otimes i d_{c}$ can be rewritten as $t_{0} \otimes t_{1} ; c_{c, c} ; i d_{c} \otimes t ; c_{c, d}$ and, therefore, if $c_{c, d}=i d$, it coincides with $\pi_{2}=t_{0} \otimes t_{1} ; c_{c, c} ; i d_{c} \otimes t$. In the same way, $\pi_{3}=t_{1} \otimes t_{0} ; i d_{c} \otimes t$ is $c_{b, a} ; t_{0} \otimes t_{1} ; c_{c, c} ; i d_{c} \otimes t$, i.e., $\pi_{2}$ if $c_{b, a}=i d$. However, as proved in this paper, such a difference is the real key to the functoriality of $Q(N)$. In particular, notice that the counterexample of Example 2.1 exploits the fact that such symmetries are identities in an essential way. Observe, in fact, the axioms of symmetric monoidal categories imply only that $t_{0} \otimes t_{1}=c_{a_{0}, a_{1}} ; t_{1} \otimes t_{0} ; c_{b_{1}, b_{0}}$. It is precisely the first axiom in Definition 1.5 that allows us to drop the symmetries and conclude that $t_{0} \otimes t_{1}=t_{1} \otimes t_{0}$, yielding an absurd situation.

\section{Conclusions}

In this paper we studied the issue of functoriality for the categorical/algebraic viewpoint of Petri net processes introduced in (Degano et al. 1996). We gave a negative result showing that no naive modification of $P(N)$ can be functorial. Then, we introduced the strongly concatenable processes as a minimal modification of concatenable processes which takes such a result into account, and showed that the construction of the strongly concatenable processes can be expressed via a 
functor $Q\left({ }_{-}\right)$. This supports the informal claim that strongly concatenable processes are the least extension of concatenable processes which yields functoriality, i.e., the least extension of GoltzReisig processes which yields an operation of concatenation and admits a functorial treatment. In addition, the paper proposed SPetriCat as an axiomatization of the category of (categories of) net behaviours; the appropriateness of such a category to the purpose has been proved by showing that $Q(-)$ embeds coreflectively Petri in SPetriCat.

The choice of the category of Petri nets studied in the paper, namely Petri exactly as defined in (Meseguer and Montanari 1990) and used in (Degano et al. 1996), has been suggested by the existence of the open problem of functoriality of the process semantics. It is worth remarking, however, that such a category is rather general, in the precise sense of allowing all the reasonable morphisms, as introduced in (Winskel 1984; Winskel 1987), which map transitions to transitions. Nevertheless, more general kinds of morphisms, e.g., mapping transitions to computations, have been occasionally proposed in the literature (Winskel 1987; Meseguer and Montanari 1990). A question which may be worth investigating in the future concerns the categorical axiomatizations of the behaviour of nets, analogous to the one presented here, when such morphisms are considered. More generally, the results of this paper might provide both the motivations and the technical ground for investigating new notions of morphism for Petri nets making $Q$ (_) a functor to SSMC, rather than a pseudo-functor.

We defined SPetriCat via a quotient which accounts precisely for the 'strings as representatives of multisets' paradigm that we pursued in this paper. We would like to remark that an equivalent, 'quotient-free', exposition of our results can be given in terms of 2-categories (more precisely, groupoid-enriched categories), the information about multisets being carried by the 2-structure. In fact, let 2-SPetriCat be the 2-category whose objects are the symmetric Petri categories, whose arrows are transition-respecting symmetric strict monoidal functors, and such that there is a 2-cell between $F$ and $G$ if there exists a monoidal natural isomorphism $F \dot{\rightarrow} G$ whose components are all symmetries. Then, considering Petri as a (trivial) 2-category, we have that $\mathcal{N}\left(\right.$ (-): 2-SPetriCat $\rightarrow$ Petri is a 2-functor, $Q\left({ }_{-}\right)$: Petri $\rightarrow 2$-SPetriCat is a pseudo 2-functor, and $Q(-) \dashv \mathcal{N}(-)$ is a pseudo coreflection. The latter means precisely that the natural family of isomorphisms between homsets which defines an adjunction is relaxed to a pseudo natural, i.e., natural up to isomorphism, family of equivalences between homcats.

We think that this alternative formulation of our axiomatization of net behaviours has some intrinsic value and deserves consideration. In fact, the pseudo functoriality expressible in 2-

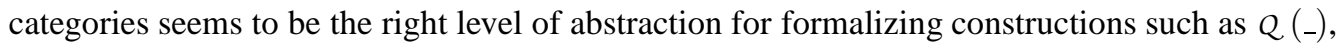
which are essentially functorial but cannot reasonably be expected to be strictly so. Nevertheless, in this paper we followed a '1-categorical' presentation, since it relies on simpler categorical tools whose use is by now rather common in our community. 


\section{References}

J. BÉNABou (1963), Categories with Multiplication. Comptes Rendue Académie Science Paris 256, 18871890.

E. Best And R. Devillers (1987), Sequential and Concurrent Behaviour in Petri Net Theory. Theoretical Computer Science 55, 87-136.

C. Brown AND D. GurR (1990), A Categorical Linear Framework for Petri Nets. In Proceedings of the 5 th LICS Symposium 208-218, IEEE Press.

C. BRown, D. GURR, AND V. DE PAIVA (1991), A Linear Specification Language for Petri Nets. Technical Report DAIMI PB-363, Computer Science Department, University of Aarhus.

W. BURNSIDE (1911), Theory of Groups of Finite Order, Cambridge University Press.

P. Degano, J. Meseguer, And U. Montanari (1996), Axiomatizing the Algebra of Net Computations and Processes. Acta Informatica 33, 641-667.

S. Eilenberg And G.M. Kelly (1966), Closed Categories. In Proceedings of the Conference on Categorical Algebra, La Jolla 1965, S. Eilenberg et al. (Eds.), 421-562, Springer-Verlag.

U. Goltz AND W. Reisig (1983), The Non-Sequential Behaviour of Petri Nets. Information and Computation 57, 125-147, Academic Press.

G.M. Kelly (1964), On MacLane's Conditions for Coherence of Natural Associativities, Commutativities, etc. Journal of Algebra 1, 397-402.

S. MacLane (1963), Natural Associativity and Commutativity. Rice University Studies 49, 28-46.

S. MACLANe (1971), Categories for the Working Mathematician. Springer-Verlag.

J. Meseguer And U. Montanari (1990), Petri Nets are Monoids. Information and Computation 88, $105^{-154}$.

E.H. MOORE (1897), Concerning the abstract group of order $k$ ! isomorphic with the symmetric substitution group on $k$ letters. Proceedings of the London Mathematical Society 28, 357-366.

M. Nielsen, G. Plotkin, And G. Winskel (1981), Petri Nets, Event Structures and Domains, Part 1. Theoretical Computer Science 13, 85-108.

C.A. Petri (1962), Kommunikation mit Automaten. PhD thesis, Institut für Instrumentelle Mathematik, Germany.

C.A. Petri (1973), Concepts of Net Theory. In Proceedings of MFCS '73, 137-146, Mathematics Institute of the Slovak Academy of Science.

C.A. Petri (1977), Non-Sequential Processes. Interner Bericht ISF-77-5, Gesellschaft für Mathematik und Datenverarbeitung, Germany.

W. REISIG (1985), Petri Nets. Springer-Verlag.

V. SASsone (1994), On the Semantics of Petri Nets: Processes, Unfoldings, and Infinite Computations. PhD Thesis TD 6/94, Dipartimento di Informatica, Università di Pisa.

V. SASSONE (1995), On the Category of Petri Net Computations. In Proceedings of TAPSOFT '95, P. Mosses et al. (Eds.), Lecture Notes in Computer Science 915, 334-348, Springer-Verlag.

V. SAssone (1996), An Axiomatization of the Algebra of Petri Net Concatenable Processes. Theoretical Computer Science 1 70, 277-296.

G. WINSKEL (1984), A New Definition of Morphism on Petri Nets. In Proceedings of STACS ' 84 , M. Fontet and K. Mehlhorn (Eds.), Lecture Notes in Computer Science 166, 140-150, Springer-Verlag.

G. Winskel (1987), Petri Nets, Algebras, Morphisms and Compositionality. Information and Computation $72,197-238$. 


\section{A. Symmetric Strict Monoidal Categories}

A symmetric strict monoidal category (Bénabou 1963; Eilenberg and Kelly 1966; MacLane 1971) is a category $\mathrm{C}$ together with a functor $\otimes: \mathrm{C} \times \mathrm{C} \rightarrow \mathrm{C}$, called the tensor product, and a selected object $e \in \mathrm{C}$, the unit object, such that $\otimes$, when viewed as a pair of operations respectively on objects and arrows of $\mathrm{C}$, forms two monoids whose units are $e$ and $i d_{e}$, and together with a family of arrows $\gamma_{x, y}: x \otimes y \rightarrow y \otimes x$, for $x$ and $y$ objects of C, such that, for each $f: x \rightarrow y$ and $g: x^{\prime} \rightarrow y^{\prime}$ in C,

$$
\begin{aligned}
\left(i d_{y} \otimes \gamma_{x, z}\right) \circ\left(\gamma_{x, y} \otimes i d_{z}\right) & =\gamma_{x, y \otimes z} \\
(g \otimes f) \circ \gamma_{x, y} & =\gamma_{x^{\prime}, y^{\prime}} \circ(f \otimes g) ; \\
\gamma_{y, x} \circ \gamma_{x, y} & =i d_{x \otimes y}
\end{aligned}
$$

Notice that the equations above mean, respectively, that $\gamma$ satisfies the relevant Kelly-MacLane (MacLane 1963; Kelly 1964) coherence axiom, that $\gamma=\left\{\gamma_{x, y}\right\}_{x, y \in C}$ is a natural transformation $\otimes \dot{\rightarrow} \otimes \circ \Delta$, where $\Delta$ is the endofunctor on $C \times C$ which 'swaps' its arguments, and that $\gamma_{x, y}$ is an isomorphism with inverse $\gamma_{y, x}$. The role of $\gamma$ is to express the commutativity 'up to isomorphism' of the structure by giving explicitly the isomorphism, e.g., between $x \otimes y$ and $y \otimes x$. Then, the axioms above guarantee the reasonable requirement that between any two functors built using exclusively $\otimes, e$, and permutations of arguments, there is at most one natural transformation composed out of such structural isomorphism, i.e., they guarantee the coherence of the structural isomorphism $\gamma$.

Theorem (MacLane 1963; Kelly 1964). Every diagram of natural transformations each arrow of which is obtained by repeatedly applying $\otimes$ to 'instances' of $\gamma$ and identities, where in turn 'instances' means components of the natural transformation at objects of $\mathrm{C}$ obtained by repeated applications of $\otimes$ to $e$ and to 'variables', commutes.

A symmetry in a symmetric monoidal category is any arrow obtained as composition and tensor of 'instances' of $\gamma$ and identities. We write Sym $_{\mathrm{C}}$ to denote the subcategory of a symmetric monoidal category C whose objects are those of $\mathrm{C}$ and whose arrows are the symmetries of $\mathrm{C}$.

A symmetric strict monoidal functor from $(\mathrm{C}, \otimes, e, \gamma)$ to $\left(\mathrm{D}, \otimes^{\prime}, e^{\prime}, \gamma^{\prime}\right)$ is a functor $\mathrm{F}: \mathrm{C} \rightarrow \mathrm{D}$ such that

$$
\begin{aligned}
\mathrm{F}(e) & =e^{\prime}, \\
\mathrm{F}(x \otimes y) & =\mathrm{F}(x) \otimes^{\prime} \mathrm{F}(y), \\
\mathrm{F}\left(\gamma_{x, y}\right) & =\gamma_{\mathrm{F} x, \mathrm{~F} y}^{\prime} .
\end{aligned}
$$

These data define the category SSMC of symmetric strict monoidal (small) categories and symmetric strict monoidal functors.

Given the symmetric strict monoidal categories $C$ and $D$ and the symmetric strict monoidal functors $\mathrm{F}: \mathrm{C} \rightarrow \mathrm{D}$ and $\mathrm{G}: \mathrm{C} \rightarrow \mathrm{D}$, a monoidal transformation from $\mathrm{F}$ to $\mathrm{G}$ is a natural transformation $\sigma: \mathrm{F} \doteq \mathrm{G}$ such that

$$
\begin{aligned}
\sigma_{e} & =i d_{e^{\prime}}, \\
\sigma_{u \otimes v} & =\sigma_{u} \otimes^{\prime} \sigma_{v} .
\end{aligned}
$$

Given a (symmetric monoidal) category $C$ and a family $\mathcal{R}$ of binary relations on the homsets of $C$ (in particular a set of equations $\mathcal{E}$ on parallel arrows of $C$ ) the (monoidal) quotient of $C$ modulo $\mathcal{R}$, is the category $C / R$, whose objects are those of $C$ and whose arrows are the equivalence classes of the arrows of $\mathrm{C}$ modulo the least equivalence closed with respect to arrow composition (and tensor product) which contains $R$. 